\title{
PSEUDO DIFFERENTIAL OPERATORS ON LOCAL HARDY SPACES ON CHÉBLI-TRIMÈCHE HYPERGROUPS
}

\author{
WALTER R. BLOOM and ZENGFU XU
}

(Received 10 July 1998; revised 15 April 1999)

Communicated by A. H. Dooley

\begin{abstract}
In this paper we consider pseudo differential operators on local Hardy spaces $\mathbf{h}^{\mathbf{p}}(0<p \leq 1)$ on Chébli-Trimèche hypergroups of exponential growth.

1991 Mathematics subject classification (Amer. Math. Soc.): primary 43A62; secondary 43A15, 43A32. Keywords and phrases: Pseudo differential operator, Hardy space, hypergroup.

Since the introduction of the Hörmander classes $L_{\eta, \delta}^{m}$ of pseudo differential operators, considerable effort has been made to establish the continuity of these on both Lebesgue spaces $L^{p}(p>1)$ and local Hardy spaces $\mathbf{h}^{\mathbf{P}}(0<p \leq 1)$ for the Euclidean space $\mathbb{R}^{n}$ (see $[\mathrm{H}, \mathrm{CV}, \mathrm{F}, \mathrm{G}, \mathrm{PS}]$ ). Recall that a pseudo differential operator on $\mathbb{R}^{n}$ is defined by

$$
T f(x)=p(x, D) f(x):=\int_{\mathbb{R}^{n}} e^{i x \xi} p(x, \xi) F_{0} f(\xi) d \xi
$$

where $F_{0}$ is the Fourier transform on $\mathbb{R}^{n}$ and $p(x, \xi)$ is called a symbol. A symbol $p(x, \xi)$ is said to belong to the class $S_{\eta, \delta}^{m}(0 \leq \eta, \delta \leq 1, m \in \mathbb{R})$ if for all multi-indices $\alpha$ and $\beta$ there exists a constant $C_{\alpha, \beta}>0$ such that

$$
\left|\frac{\partial^{\alpha}}{\partial \xi^{\alpha}} \frac{\partial^{\beta}}{\partial x^{\beta}} p(x, \xi)\right| \leq C_{\alpha, \beta}(1+|\xi|)^{m-\eta|\alpha|+\delta|\beta|} .
$$

The operator $p(\cdot, D)$ is said to belong to the class $L_{\eta, \delta}^{m}$ if $p(x, \xi) \in S_{\eta, \delta}^{m}$. In [H] Hörmander proved among other things that the operators in $L_{\eta, \delta}^{0}(0 \leq \delta<\eta \leq 1)$ are bounded on $L^{2}$. Calderón and Vaillancourt in [CV] extended this result to the case $0 \leq \delta=\eta<1$. Fefferman [F] gave a different extension of Hörmander's result and showed that the operators in $L_{\eta, \delta}^{-\mu}(0 \leq \delta<\eta \leq 1, \mu \geq n|1 / p-1 / 2|(1-\eta))$ map
\end{abstract}

(c) 2000 Australian Mathematical Society $0263-6115 / 2000 \$ A 2.00+0.00$ 
$L^{p}$ into itself boundedly for $1<p<\infty$. The continuity results concerning these operators in the local Hardy space $\mathbf{h}^{\mathbf{p}}$ were discussed in [PS] and [G]. The $L^{1}-L^{1}$, $L^{2}-L^{2}$ and $h^{1}-L^{1}$-boundedness of certain pseudo-differential operators were also investigated in $[\mathrm{K}]$ in the setting of noncompact symmetric spaces.

In this paper we study $\mathbf{h}^{\mathbf{p}}$-boundedness for pseudo differential operators in the classes $L_{\eta, \delta}^{m}$ on Chébli-Trimèche hypergroups with exponential growth. In Section 1 we collect some basic facts of harmonic analysis on these hypergroups. We then (Section 2) introduce pseudo differential operators on Chébli-Trimèche hypergroups and prove $L^{2}$-boundedness for operators in certain classes $L_{\eta, \delta}^{m}$. Finally, in Section 3 we establish the continuity result for operators on the local Hardy spaces $\mathbf{h}^{\mathbf{p}}(0<p \leq 1)$ using the atomic and molecular decomposition of $\mathbf{h}^{\mathbf{p}}$. It turns out that the extra restriction of holomorphy on the symbols $p(x, \xi)$ with respect to $\xi$ is necessary for the operators to be bounded on $\mathbf{h}^{\mathbf{p}}$ when the volumes of balls grow exponentially.

Because of the exponential volume growth and the generalized convolution on the hypergroup, the standard methods do not apply. Many basic facts relying on the structure of a Euclidean space are largely unavailable; the Fourier transform on hypergroups is far less well understood than on Euclidean spaces. We employ techniques for noncompact symmetric spaces to overcome the difficulties caused by the exponential growth, and use properties of the Fourier transform and estimates for the characters to handle the problems arising from the generalized translation. Our method is a combination of techniques for Euclidean spaces and for noncompact symmetric spaces, and indeed the approach used here can be easily applied to noncompact symmetric spaces.

\section{Harmonic analysis on Chébli-Trimèche hypergroups}

Chébli-Trimèche hypergroups (see Definition 1.1 below) form a class of 'onedimensional' hypergroups on $\mathbb{R}_{+}$arising from Sturm-Liouville boundary value problems where the solutions coincide with the characters of the hypergroup in question. The convolution structure of the hypergroup is related to the following second order differential operator:

$$
L=L_{A, x}:=-\frac{d^{2}}{d x^{2}}-\frac{A^{\prime}(x)}{A(x)} \frac{d}{d x}
$$

where the function $A$ is continuous on $\mathbb{R}_{+}$, twice continuously differentiable on $\left.\mathbb{R}_{+}^{*}=\right] 0, \infty[$ and satisfies the following conditions:

(1.2) $A(0)=0$ and $A(x)>0$ for $x>0$;

(1.3) $A$ is increasing and unbounded;

(1.4) $A^{\prime}(x) / A(x)=(2 \alpha+1) / x+B(x)$ on a neighbourhood of 0 where $\alpha>-1 / 2$ and $B$ is an odd $C^{\infty}$-function on $\mathbb{R}$; 
(1.5) $A^{\prime}(x) / A(x)$ is a decreasing $C^{\infty}$-function on $\mathbb{R}_{+}^{*}$ and hence $\rho:=1 / 2 \lim _{x \rightarrow+\infty}$ $A^{\prime}(x) / A(x) \geq 0$ exists.

Such a function $A$ is called a Chébli-Trimèche function (see [BH, Section 3.5]).

DEFINITION 1.1. A hypergroup $\left(\mathbb{R}_{+}, *\right)$ is called a Chébli-Trimèche hypergroup if there exists a Chébli-Trimèche function $A$ such that for any real-valued function $f$ on $\mathbb{B}_{+}$that is the restriction of an even nonnegative $C^{\infty}$-function on $\mathbb{R}$ the generalized translation $u(x, y)=T_{x} f(y)$ (see (1.9) below) is the solution of the following Cauchy problem:

$$
\left\{\begin{array}{l}
\left(L_{A, x}-L_{A, y}\right) u(x, y)=0, \\
u(x, 0)=f(x), \quad u_{y}(x, 0)=0, \quad x>0 .
\end{array}\right.
$$

We denote by $\left(\mathbb{R}_{+}, *(A)\right)$ the Chébli-Trimèche hypergroup associated with $A$.

REMARK. In particular, if the function $A$ is of the form $A(x):=x^{2 \alpha+1}$ with $\alpha>-1 / 2$ then $\left(\mathbb{R}_{+}, *(A)\right)$ is a Bessel-Kingman hypergroup, and if $A(x):=$ $\sinh ^{2 \alpha+1} x \cosh ^{2 \beta+1} x$ with $\alpha \geq \beta \geq-1 / 2$ and $\alpha \neq-1 / 2$ then $\left(\mathbb{R}_{+}, *(A)\right)$ is a Jacobi hypergroup.

The hypergroup $\left(\mathbb{R}_{+}, *(A)\right)$ is noncompact and commutative with neutral element 0 and the identity mapping as the involution. Haar measure on $\left(\mathbb{R}_{+}, *(A)\right)$ is given by $\omega:=A \lambda_{\mathbb{R}_{+}}$where $\lambda_{\mathbb{R}_{+}}$is the usual Lebesgue measure on $\mathbb{R}_{+}$. For an $\omega$-measurable subset $E$ we denote by $|E|$ its Haar measure and $\chi_{E}$ its characteristic function. For $x_{0} \in \mathbb{R}_{+}$and $r>0$ we denote by $B\left(x_{0}, r\right)$ the open interval $] \max \left\{0, x_{0}-r\right\}, x_{0}+r[$. The growth of the hypergroup is determined by the number $\rho$ in (1.5). For the hypergroup to be of exponential growth it is necessary and sufficient that $\rho>0$ (see $\left[\mathrm{BH}\right.$, Proposition 3.5.55]), for then (1.5) implies that $A(x) \geq A(1) e^{2 \rho(x-1)}$ for $x \geq 1$. Otherwise we say that the hypergroup is of subexponential growth (which includes polynomial growth). In the sequel we only consider hypergroups of exponential growth.

The multiplicative functions on $\left(\mathbb{R}_{+}, *(A)\right)$ coincide with the solutions $\varphi_{\lambda}(\lambda \in \mathbb{C})$ of the differential equation

$$
L \varphi_{\lambda}(x)=\left(\lambda^{2}+\rho^{2}\right) \varphi_{\lambda}(x), \quad \varphi_{\lambda}(0)=1, \quad \varphi_{\lambda}^{\prime}(0)=0
$$

and the dual space $\mathbb{R}_{+}^{\wedge}$ can be identified with the parameter set $\mathbb{R}_{+} \cup i[0, \rho]$.

For $0<p \leq \infty$ the Lebesgue space $L^{p}\left(\mathbb{R}_{+}, A d x\right)$ is defined as usual, and we denote by $\|f\|_{p, A}$ the $L^{p}$-norm of $f \in L^{p}\left(\mathbb{R}_{+}, A d x\right)$. For $f \in L^{1}\left(\mathbb{R}_{+}, A d x\right)$ the Fourier transform of $f$ is given by

$$
\widehat{f}(\lambda):=\int_{\mathbb{R}_{+}} f(x) \varphi_{\lambda}(x) A(x) d x .
$$


THEOREM 1.1 (Levitan-Plancherel; see [BH, Theorem 2.2.13]). There exists a unique non-negative measure $\pi$ on $\mathbb{R}_{+}^{\wedge}$ with support $[0, \infty[$ such that the Fourier transform induces an isometric isomorphism from $L^{2}\left(\mathbb{R}_{+}, A d x\right)$ onto $L^{2}\left(\mathbb{R}_{+}^{\wedge}, \pi\right)$, and for any $f \in L^{1} \cap L^{2}\left(\mathbb{R}_{+}, A d x\right)$

$$
\int_{\mathbb{R}_{+}}|f(x)|^{2} A(x) d x=\int_{\mathbb{R}_{+}^{\hat{T}}}|\widehat{f}(\lambda)|^{2} \pi(d \lambda) .
$$

The inverse is given by

$$
f(x)=\int_{\mathbb{R}_{+}^{+}} \widehat{f}(\lambda) \varphi_{\lambda}(x) \pi(d \lambda) .
$$

To determine the Plancherel measure $\pi$ we must place a further (growth) restriction on $A$. We assume that $A$ has the form $A(x)=x^{2 \alpha+1} C(x)$ where $C(x)$ is an even $C^{\infty}$-function on $\mathbb{R}$ satisfying

(1.7) there exists a constant $\epsilon>0$ such that for all $x \in\left[x_{0}, \infty\left[, x_{0}>0\right.\right.$

$$
\frac{C^{\prime}(x)}{C(x)}=2 \rho-\frac{2 \alpha+1}{x}+e^{-\epsilon x} D(x)
$$

with $D$ being a $C^{\infty}$-function bounded together with its derivatives (see [T2]).

We also assume that $\alpha \geq 0$ and for each $k \in \mathbb{N},\left(A^{\prime}(x) / A(x)\right)^{(k)}$ is bounded for large $x \in \mathbb{R}_{+}$.

THEOREM 1.2 (see [BX1, Proposition 3.17]). The Plancherel measure $\pi$ is absolutely continuous with respect to Lebesgue measure and has density $|c(\lambda)|^{-2}$ where the function $c$ satisfies the following:

(i) $c(-\lambda)=\overline{c(\lambda)}$;

(ii) The function $|c(\lambda)|^{-2}$ is continuous on $\mathbb{R}_{+}$and there exist positive constants $C_{1}, C_{2}, K$ such that for any $\lambda \in \mathbb{C}$

$$
\begin{aligned}
C_{1}|\lambda|^{2} & \leq|c(\lambda)|^{-2} \leq C_{2}|\lambda|^{2}, & & |\lambda| \leq K ; \\
C_{1}|\lambda|^{2 \alpha+1} & \leq|c(\lambda)|^{-2} \leq C_{2}|\lambda|^{2 \alpha+1}, & & |\lambda|>K .
\end{aligned}
$$

The following result can be found in [BX1, Lemma 2.5 and Lemma 3.28].

LEMMA 1.1. We have

$$
A(x) \sim x^{2 \alpha+1} \quad\left(x \rightarrow 0^{+}\right)
$$

and

$$
A(x) \sim e^{2 \rho x} \quad(x \rightarrow+\infty)
$$


Let $\epsilon_{x}$ be the unit point mass at $x \in \mathbb{R}_{+}$. For any $x, y \in \mathbb{R}_{+}$the probability measure $\epsilon_{x} * \epsilon_{y}$ is $\omega$-absolutely continuous with

$$
\operatorname{supp}\left(\epsilon_{x} * \epsilon_{y}\right) \subset[|x-y|, x+y] .
$$

We denote by $T_{x} f$ the generalized translation of a function $f$ by $x \in \mathbb{R}_{+}$defined by

$$
T_{x} f(y):=\int_{\mathbb{R}_{+}} f(z)\left(\epsilon_{x} * \epsilon_{y}\right)(d z) .
$$

The convolution of two functions $f$ and $g$ is defined by

$$
f * g(x)=\int_{\mathbb{R}_{+}} T_{x} f(y) g(y) A(y) d y .
$$

We now introduce Schwartz functions and distributions on the hypergroup (see [BX3]). For $0<p \leq 2$ the generalized Schwartz space $\mathscr{S}_{p}\left(\mathbb{R}_{+}, *(A)\right)$ consists of the restrictions to $\mathbb{R}_{+}$of all functions in $\mathscr{S}_{p}(\mathbb{R})$ where

$$
\mathscr{S}_{p}(\mathbb{R}):=\left\{g \in C^{\infty}(\mathbb{R}): g \text { is even and } \mu_{k . l}^{p}(g)<\infty, k, l \in \mathbb{N}_{0}\right\}
$$

and

$$
\mu_{k, l}^{p}(g):=\sup _{x \in \mathbb{R}_{+}}(1+x)^{l} \varphi_{0}(x)^{-2 / p}\left|g^{(k)}(x)\right|
$$

For $0<p \leq 2$ set

$$
\mathscr{F}_{\sigma}:=\{z \in \mathbb{C}:|\operatorname{Im}(z)| \leq \sigma \rho\}
$$

with $\sigma=2 / p-1$ and $\rho>0$ as in (1.5). Let $\mathscr{S}\left(\mathscr{F}_{\sigma}\right)$ denote the extended Schwartz space defined by all functions $h$ that are even and holomorphic in the interior of $\mathscr{F}_{\sigma}$, and such that $h$ together with all its derivatives extend continuously to $\mathscr{F}_{\sigma}$ and satisfy $\sup _{\lambda \in \mathscr{F}_{\sigma}}\left|h^{(k)}(\lambda)\right|<\infty$ for any $k, l \in \mathbb{N}$. Also, we denote by $\mathscr{S}_{\epsilon \rho}\left(\mathbb{R}_{+}\right)$the space of the restrictions to $\mathbb{R}_{+}$of the functions in $\mathscr{S}_{\epsilon \rho}(\mathbb{R})$ where

$$
\mathscr{S}_{\epsilon \rho}(\mathbb{R})=\left\{g \in C^{\infty}(\mathbb{R}): g \text { is even and } v_{k, l}^{(\epsilon)}(g)<\infty\right\}
$$

with

$$
v_{k, l}^{(\epsilon)}(g):=\sup _{t \in \mathbb{R}_{+}}(1+t)^{l} e^{\epsilon \rho t}\left|g^{(k)}(t)\right| .
$$

Note that $\mathscr{S}_{0}=\mathscr{S}\left(\mathscr{F}_{0}\right)$ is the usual Schwartz space on $\mathbb{R}_{+}$and will be denoted by $\mathscr{S}\left(\mathbb{R}_{+}\right)$. In the sequel we use $F$ to denote the Fourier transform on the hypergroup, $F_{0}$ the classical Fourier transform and $\mathscr{A}$ the Abel transform (see [T1] for the definition of this last transform). 
THEOREM 1.3 (see [BX3])). Let $\sigma=2 / p-1$ with $0<p \leq 2$. Then the Fourier transform $F$ on $\left(\mathbb{R}_{+}, *(A)\right)$ is an isomorphism from $\mathscr{S}_{p}\left(\mathbb{R}_{+}, *(A)\right)$ to $\mathscr{S}\left(\mathscr{F}_{\delta}\right)$, the classical Fourier $F_{0}$ an isomorphism from $\mathscr{S}_{\epsilon \rho}\left(\mathbb{R}_{+}\right)$to $\mathscr{S}\left(\mathscr{F}_{\delta}\right)$, and the Abel transform $\mathscr{A}$ an isomorphism from $\mathscr{S}_{p}\left(\mathbb{R}_{+}, *(A)\right)$ to $\mathscr{S}_{\epsilon \rho}\left(\mathbb{R}_{+}\right)$satisfying $F f=F_{0}(\mathscr{A} f)$.

A $p$-distribution on $\mathbb{R}_{+}$is a continuous linear functional on $\mathscr{S}_{p}\left(\mathbb{R}_{+}, *(A)\right)$; the totality of $p$-distributions on $\mathbb{R}_{+}$is denoted by $\mathscr{S}_{p}^{\prime}\left(\mathbb{R}_{+}, *(A)\right)$. We define the Fourier transform of $f \in \mathscr{S}_{p}^{\prime}\left(\mathbb{R}_{+}, *(A)\right)$ by

$$
\widehat{f}(\phi):=f\left(F^{-1} \phi\right), \quad \phi \in \mathscr{S}\left(\mathscr{F}_{\sigma}\right) .
$$

By Theorem 1.3, $\widehat{f}$ is well defined as a distribution in $\mathscr{S}^{\prime}\left(\mathscr{F}_{\sigma}\right)$ and $F$ is continuous on $\mathscr{S}_{p}\left(\mathbb{R}_{+}, *(A)\right)$. The convolution of $f \in \mathscr{S}_{p}^{\prime}\left(\mathbb{R}_{+}, *(A)\right)$ and $\phi \in \mathscr{S}_{p}\left(\mathbb{R}_{+}, *(A)\right)$ is a $p$-distribution defined by

$$
f * \phi(\psi):=f(\phi * \psi), \quad \psi \in \mathscr{S}_{p}\left(\mathbb{R}_{+}, *(A)\right)
$$

We now give some useful estimates for characters and their derivatives.

Lemma 1.2 (see [BX3, Lemma 3.4]). Let $\lambda=\xi+i \eta \in \mathbb{C}$. Then

(i) $\left|\varphi_{\lambda}(x)\right| \leq e^{|\eta| x} \varphi_{0}(x)$;

(ii) $e^{-\rho x} \leq \varphi_{0}(x) \leq C(1+x) e^{-\rho x}$;

(iii) for any $k \in \mathbb{N}_{0}$ we have

$$
\left|\frac{\partial^{k}}{\partial \lambda^{k}} \varphi_{\lambda}(x)\right| \leq x^{k} e^{|\eta| x} \varphi_{0}(x) .
$$

LEMMA 1.3 (see $[\mathrm{BX} 4])$. Let $\lambda=\xi+i \eta \in \mathbb{C}$ and $k \in \mathbb{N}_{0}$. Then

$$
\left|\varphi_{\lambda}^{(k)}(x)\right| \leq \begin{cases}C_{A}(1+|\lambda|)^{k} e^{|\eta| x} & |\lambda| x \leq 1, x \leq 1 \\ C_{A} x A(x)^{-1 / 2} e^{|\eta| x} & |\lambda| x \leq 1, x>1 \\ C_{A} A(x)^{-1 / 2}|c(\lambda)|(1+|\lambda|)^{k} e^{|\eta| x} & |\lambda| x>1\end{cases}
$$

We also have the following alternative estimate:

$$
\left|\varphi_{\lambda}^{(k)}(x)\right| \leq C_{A} A(x)^{-1 / 2}(|\lambda| x)^{1 / 2-a}|c(\lambda)|(1+|\lambda|)^{k} e^{|\eta| x}, \quad|\lambda| x \leq 1, x>1 .
$$

Finally, we shall use $C$ to denote a positive constant which value may vary from line to line. Dependence of such constants upon parameters of interest will be indicated through the use of subscripts. 


\section{Continuity of pseudo differential operators in $L^{2}\left(\mathbb{R}_{+}, A d x\right)$}

In this section we introduce Hörmander's class $L_{\eta, \delta}^{m}$ of pseudo differential operators on Chébli-Trimèche hypergroups and investigate their continuity properties with respect to the $L^{2}$ norm.

DEFINITION 2.1. A pseudo differential operator $p(x, D)$ on $\left(\mathbb{R}_{+}, *(A)\right)$ is defined by

$$
T f(x)=p(x, D) f(x):=\int_{0}^{\infty} \widehat{f}(\lambda) p(x, \lambda) \varphi_{\lambda}(x) \pi(d \lambda), \quad x \in \mathbb{R}_{+}
$$

where $p(x, \lambda)$ is an even function with respect to both $x$ and $\lambda$ on $\mathbb{R} \times \mathbb{C}$ and is called a symbol. A symbol $p(x, \lambda)$ is said to belong to the class $S_{\eta, \delta}^{m}(0 \leq \eta, \delta \leq 1, m \in \mathbb{R})$ if for all nonnegative integers $k, l$ there exists a constant $C_{k l}>0$ such that

$$
\left|\frac{\partial^{k}}{\partial \lambda^{k}} \frac{\partial^{l}}{\partial x^{l}} p(x, \lambda)\right| \leq C_{k l}(1+|\lambda|)^{m-\eta k+\delta l} .
$$

The operator $p(x, D)$ is said to belong to the class $L_{\eta, \delta}^{m}$ if $p(x, \lambda) \in S_{\eta, \delta}^{m}$.

To obtain an $L^{2}$ estimate we relate $p(x, D)$ to a pseudo differential operator on $\mathbb{R}$. For $x>0$ we put

$$
G(x):=\frac{1}{4}\left(\frac{A^{\prime}(x)}{A(x)}\right)^{2}+\frac{1}{2}\left(\frac{A^{\prime}(x)}{A(x)}\right)^{\prime}-\rho^{2} .
$$

Recall that for each $\lambda \in \mathbb{C}, \lambda \neq 0$ the differential equation

$$
L u=\left(\lambda^{2}+\rho^{2}\right) u
$$

has two linearly independent solutions $\Phi_{\lambda}$ and $\Phi_{-\lambda}$ over ]0, $\infty$ [ satisfying (see [BX1, Section 3])

$$
\varphi_{\lambda}(x)=c(\lambda) \Phi_{\lambda}(x)+c(-\lambda) \Phi_{-\lambda}(x), \quad x>0
$$

and

$$
\Phi_{-\lambda}(x)=A(x)^{-1 / 2} e^{-i \lambda x}(1+h(x, \lambda)), \quad x>0, \quad \operatorname{Im}(\lambda) \leq 0
$$

where

$$
h(x, \lambda)=\sum_{j=0}^{\infty} \widetilde{h}_{j}(x, \lambda)
$$


and

$$
\left\{\begin{array}{l}
\tilde{h}_{j}(x, \lambda)=h_{j+1}(x, \lambda)-h_{j}(x, \lambda) \\
h_{0}(x, \lambda)=0 \\
h_{j}(x, \lambda)=\frac{1}{2 i \lambda} \int_{x}^{\infty}\left(1-e^{2 i \lambda(x-t)}\right) G(t)\left(1+h_{j}(t, \lambda)\right) d t, \quad j \in \mathbb{N}_{0} .
\end{array}\right.
$$

We have the following estimates for $h(x, \lambda)$ and its derivatives.

LEMMA 2.1. For any nonnegative integers $k, l$,

$$
\left|\frac{\partial^{k}}{\partial \lambda^{k}} \frac{\partial^{l}}{\partial x^{l}} h(x, \lambda)\right| \leq C_{A, k l}(1+|\lambda|)^{-k-1} e^{-\epsilon x}, \quad x>1, \lambda>0
$$

where $\epsilon$ is as in (1.7).

PROOF. In view of (1.7) and (2.1) we observe that

$$
\left|G^{(k)}(x)\right| \leq C_{A, k} e^{-\epsilon x}, \quad x>1, k=0,1,2, \ldots
$$

By (2.5) we have

$$
\left\{\begin{array}{l}
\widetilde{h}_{0}(x, \lambda)=\frac{1}{2 i \lambda} \int_{x}^{\infty}\left(1-e^{2 i \lambda(x-t)}\right) G(t) d t \\
\widetilde{h}_{j+1}(x, \lambda)=\frac{1}{2 i \lambda} \int_{x}^{\infty}\left(1-e^{2 i \lambda(x-t)}\right) G(t) \widetilde{h}_{j}(t, \lambda) d t, \quad j \in \mathbb{N}_{0} .
\end{array}\right.
$$

Let $\Phi(x)=\int_{x}^{\infty} G(t) d t(x>1)$. Then integrating by parts gives

$$
\tilde{h}_{0}(x, \lambda)=\int_{x}^{\infty} e^{2 i \lambda(x-t)} \Phi(t) d t, \quad x>1, \lambda>0
$$

and

$$
\begin{aligned}
\frac{\partial}{\partial x} \widetilde{h}_{0}(x, \lambda) & =-\Phi(x)-\int_{x}^{\infty} \Phi(t) d e^{2 i \lambda(x-t)} \\
& =\int_{x}^{\infty} e^{2 i \lambda(x-t)} \Phi^{\prime}(t) d t, \quad x>1, \lambda>0 .
\end{aligned}
$$

Hence by induction we obtain for any nonnegative integer $l$

$$
\frac{\partial^{l}}{\partial x^{l}} \widetilde{h}_{0}(x, \lambda)=\int_{x}^{\infty} e^{2 i \lambda(x-t)} \Phi^{(l)}(t) d t .
$$


Thus for any nonnegative integer $k$

$$
\frac{\partial^{k}}{\partial \lambda^{k}} \frac{\partial^{l}}{\partial x^{l}} \widetilde{h}_{0}(x, \lambda)=(2 i)^{k} \int_{x}^{\infty}(x-t)^{k} e^{2 i \lambda(x-t)} \Phi^{(l)}(t) d t .
$$

By (2.6) this gives for $0<\lambda \leq 1$ and $x>1$

$$
\left|\frac{\partial^{k}}{\partial \lambda^{k}} \frac{\partial^{l}}{\partial x^{l}} \widetilde{h}_{0}(x, \lambda)\right| \leq C_{A, k l} e^{-\epsilon x} .
$$

For $\lambda>1$ we integrate by parts $k+1$ times to obtain

$$
\begin{aligned}
\frac{\partial^{k}}{\partial \lambda^{k}} \frac{\partial^{l}}{\partial x^{l}} \widetilde{h}_{0}(x, \lambda)= & \frac{(-1)^{k+1}}{2 i \lambda^{k+1}} \Phi^{(l)}(x) \\
& +\frac{(-1)^{k+1}}{2 i \lambda^{k+1}} \int_{x}^{\infty} \frac{\partial^{k+1}}{\partial t^{k+1}}\left[(x-t)^{k} \Phi^{(l)}(t)\right] e^{2 i \lambda(x-t)} d t .
\end{aligned}
$$

Therefore,

$$
\left|\frac{\partial^{k}}{\partial \lambda^{k}} \frac{\partial^{l}}{\partial x^{l}} \tilde{h}_{0}(x, \lambda)\right| \leq C_{A, k l}(1+\lambda)^{-k-1} e^{-\epsilon x}, \quad x>1, \lambda>0 .
$$

For $\tilde{h}_{1}(x, \lambda)$, using (2.7) we have

$$
\frac{\partial}{\partial x} \widetilde{h}_{1}(x, \lambda)=-\int_{x}^{\infty} e^{2 i \lambda(x-t)} G(t) \widetilde{h}_{0}(t, \lambda) d t .
$$

Now integrating by parts we have by induction

$$
\frac{\partial^{l}}{\partial x^{l}} \widetilde{h}_{1}(x, \lambda)=-\int_{x}^{\infty} e^{2 i \lambda(x-t)} \frac{\partial^{l-1}}{\partial t^{l-1}}\left(G(t) \widetilde{h}_{0}(t, \lambda)\right) d t, \quad x>1, \lambda>0 .
$$

Hence for $x>1$ and $\lambda>0$

$$
\frac{\partial^{k}}{\partial \lambda^{k}} \frac{\partial^{l}}{\partial x^{l}} \widetilde{h}_{1}(x, \lambda)=-\sum_{q=0}^{k}\left(\begin{array}{l}
k \\
q
\end{array}\right) \int_{x}^{\infty}[2 i(x-t)]^{k-q} e^{2 i \lambda(x-t)} \frac{\partial^{q}}{\partial \lambda^{q}} \frac{\partial^{l-1}}{\partial t^{l-1}}\left(G(t) \widetilde{h}_{0}(t, \lambda)\right) d t .
$$

If $\lambda>1$ then we integrate by parts $k-q+1$ times for each $q=0,1, \ldots, k$. Thus by (2.6) and (2.8) we obtain

$$
\left|\frac{\partial^{k}}{\partial \lambda^{k}} \frac{\partial^{l}}{\partial x^{l}} \widetilde{h}_{1}(x, \lambda)\right| \leq C_{A, k l}(1+\lambda)^{-k-1} e^{-2 \epsilon x}, \quad x>1, \lambda>0 .
$$

By (2.7) and induction we have $j=0,1, \ldots$

$$
\left|\frac{\partial^{k}}{\partial \lambda^{k}} \frac{\partial^{l}}{\partial x^{l}} \tilde{h}_{j}(x, \lambda)\right| \leq C_{A, k l}(1+\lambda)^{-k-1} e^{-(j+1) \epsilon x}, \quad x>1, \lambda>0 .
$$

The lemma now follows immediately from (2.4) and (2.9). 
For an even $C^{\infty}$-function $f$ on $\mathbb{R}$ and a positive integer $k$ let $L^{0} f=f$ and $L^{k} f=L\left(L^{k-1} f\right)$ where $L$ is defined by (1.1).

LEMMA 2.2. (i) $L^{k} f$ extends to an even $C^{\infty}$-function on $\mathbb{R}$.

(ii) There exist $a>0$ and $s_{k} \in \mathbb{N}_{0}$ satisfying that for each $x \in[0, a]$ and $j=0,1, \ldots, s_{k}$ there exist $\xi_{j}=\xi_{j}(x, k) \in[0, x]$ such that

$$
\left|L^{k} f(x)\right| \leq C_{A, k}\left(\sum_{i=1}^{2 k} \sum_{j=0}^{s_{k}}\left|f^{(i)}\left(\xi_{j}\right)\right|+\sum_{i=1}^{2 k}\left|f^{(i)}(x)\right|\right) .
$$

(iii) There exists $a>0$ such that

$$
\left|L^{k} f(x)\right| \leq \begin{cases}C_{A} \sum_{i=0}^{2 k-1} x^{-k}\left|f^{(2 k-i)}(x)\right|, & 0<x \leq a ; \\ C_{A} \sum_{i=0}^{2 k}\left|f^{(i)}(x)\right|, & x>a .\end{cases}
$$

Proof. Appealing to (1.1) and (1.4) there exists $a>0$ such that

$$
L f(x)=-f^{\prime \prime}(x)-\left(\frac{2 \alpha+1}{x}+B(x)\right) f^{\prime}(x), \quad 0<x \leq a .
$$

Hence by induction we obtain

$$
\left|L^{k} f(x)\right| \leq C_{A, k} \sum_{i=0}^{2 k-3} x^{-i}\left|(L f)^{(2 k-2-i)}(x)\right|, \quad 0<x \leq a
$$

and

$$
(L f)^{(k)}(x)=-f^{(k+2)}(x)-\sum_{i=0}^{k}\left(\begin{array}{l}
k \\
i
\end{array}\right)\left((-1)^{i} i ! x^{-i-1}+B^{(i)}(x)\right) f^{(k+1-i)}(x) .
$$

Consequently,

$$
\left|L^{k} f(x)\right| \leq C_{A, k} \sum_{i=0}^{2 k-1} x^{-i}\left|f^{(2 k-i)}(x)\right|, \quad 0<x \leq a .
$$

The proof of the remainder of the lemma can be found in [BX3, Lemma 4.18].

We now give $L^{2}$-estimates for a class $L_{\eta, \delta}^{m}$ of pseudo differential operators.

THEOREM 2.1. Suppose that $m \leq 0,0<\eta \leq 1$ and $p(x, \lambda) \in S_{\eta 0^{\circ}}^{m}$ Then the pseudo differential operator $p(x, D)$ is bounded on $L^{2}\left(\mathbb{R}_{+}, *(A)\right)$. 
PROOF. Let $\psi$ be an even $C^{\infty}$-function on $\mathbb{R}$ such that $\psi(x)=1$ if $|x| \leq 1$ and $\psi(x)=0$ if $|x| \geq 2$. Put $p_{1}(x, \lambda)=p(x, \lambda) \psi(x)$ and $p_{2}(x, \lambda)=p(x, \lambda)(1-\psi(x))$. Thus $p_{i}(x, \lambda) \in S_{\eta, \delta}^{m}(i=1,2)$ and, using Definition 2.1,

$$
\begin{aligned}
p(x, D) f(x) & =\int_{0}^{\infty} \widehat{f}(\lambda) p_{1}(x, \lambda) \varphi_{\lambda}(x) \pi(d \lambda)+\int_{0}^{\infty} \widehat{f}(\lambda) p_{2}(x, \lambda) \varphi_{\lambda}(x) \pi(d \lambda) \\
& =p_{1}(x, D) f(x)+p_{2}(x, D) f(x)
\end{aligned}
$$

for $f \in L^{2}\left(\mathbb{R}_{+}, *(A)\right)$.

We first prove that $p_{1}(x, D)$ is $L^{2}$-bounded. For each $\lambda>0$ let $R_{\lambda}$ be the Fourier transform of $p_{1}(\cdot, \lambda)$ on $\mathbb{R}$. Then by (1.1) and (1.6)

$$
\begin{aligned}
\left(\zeta^{2}+\rho^{2}\right)^{k} R_{\lambda}(\zeta) & =\int_{0}^{\infty} p_{1}(x, \lambda) L^{k} \varphi_{\zeta}(x) A(x) d x \\
& =\int_{0}^{\infty} L^{k} p_{1}(x, \lambda) \varphi_{\zeta}(x) A(x) d x .
\end{aligned}
$$

Hence using Lemma 1.2, Lemma 2.2 and Definition 2.1 we obtain for any nonnegative integer $k$

$$
\left|R_{\lambda}(\zeta)\right| \leq C_{A, k}(1+\lambda)^{m}(1+\zeta)^{-2 k} .
$$

In view of Theorem 1.1 we observe

$$
p_{1}(x, D) f(x)=\int_{0}^{\infty}\left(\int_{0}^{\infty} \widehat{f}(\lambda) R_{\lambda}(\zeta) \varphi_{\lambda}(x) \pi(d \lambda)\right) \varphi_{\zeta}(x) \pi(d \zeta) .
$$

Therefore by Theorem 1.1, Theorem 1.2(ii) and (2.10) with $k>\alpha+1$

$$
\begin{aligned}
\left\|p_{1}(\cdot, D) f\right\|_{2, A} & \leq C_{A} \int_{0}^{\infty}\left\|R_{\lambda}(\zeta) \widehat{f}(\lambda)\right\|_{2, \pi} \pi(d \zeta) \\
& \leq C_{A}\|\widehat{f}\|_{2, \pi} \int_{0}^{\infty}(1+\zeta)^{-2 k}|c(\lambda)|^{-2} d \lambda \\
& \leq C_{A}\|f\|_{2, A} .
\end{aligned}
$$

It remains to show that $p_{2}(x, D)$ is bounded on $L^{2}\left(\mathbb{R}_{+}, *(A)\right)$. Using Theorem 1.2, (2.2) and (2.3) we have

$$
\begin{aligned}
p_{2}(x, D) f(x) & =C_{0} \int_{-\infty}^{\infty} \widehat{f}(\lambda) p_{2}(x, \lambda) \Phi_{-\lambda}(x) c(\lambda)^{-1} d \lambda \\
& =C_{0} A(x)^{-1 / 2} \int_{-\infty}^{\infty} \widehat{f}(\lambda) c(\lambda)^{-1} p_{2}(x, \lambda)(1+h(x, \lambda)) e^{i \lambda x} d \lambda \\
& =C_{0} A(x)^{-1 / 2} \widetilde{p}_{2}(x, D) g(x)
\end{aligned}
$$


where $\tilde{p}_{2}(x, \lambda)=p_{2}(x, \lambda)(1+h(x, \lambda))$ and $F_{0} g(\lambda)=\widehat{f}(\lambda) c(\lambda)^{-1}$ is the classical Fourier transform of $g$ on $\mathbb{R}$. Now $\widetilde{p}_{2}(x, D)$ is a pseudo differential operator on $\mathbb{R}$. In view of Lemma 2.1 we see that $\widetilde{p}_{2}(x, \lambda)$ is a symbol in $S_{\eta 0}^{m}$ on $\mathbb{R}$ such that

$$
\left|\frac{\partial^{k}}{\partial \lambda^{k}} \frac{\partial^{l}}{\partial x^{l}} \widetilde{p}_{2}(x, \lambda)\right| \leq C_{k l}(1+|\lambda|)^{m-\eta k} .
$$

Hence (see $[\mathrm{F}]$ ) the operator $\widetilde{p}_{2}(x, D)$ is $L^{2}$-bounded on $\mathbb{R}$. Therefore by (2.11), Theorem 1.1 and Theorem 1.2 we have

$$
\begin{aligned}
\left\|p_{2}(x, D) f(x)\right\|_{2, A}^{2} & =C_{0} \int_{0}^{\infty}\left|\widetilde{p}_{2}(x, D) g(x)\right|^{2} d x \\
& \leq C_{A} \int_{-\infty}^{\infty}\left|\widehat{f}(\lambda) c(\lambda)^{-1}\right|^{2} d \lambda \\
& =C_{A}\|f\|_{2, A}^{2} .
\end{aligned}
$$

This completes the proof of the theorem.

\section{Pseudo differential operators on local Hardy spaces}

In this section we establish the continuity result for the class $L_{n 0}^{m}$ of pseudo differential operators on local Hardy spaces. We begin with the definition of the local Hardy space $\mathbf{h}^{\mathbf{p}}$ and its atomic and molecular decomposition characterization (see [BX4]).

For $f \in \mathscr{S}_{1}^{\prime}\left(\mathbb{R}_{+}, *(A)\right)$ the local heat maximal function is defined by

$$
H_{0}^{+} f(x):=\sup _{0<t \leq 1}\left|f * h_{t}(x)\right|
$$

where $h_{t}$ is the heat kernel (see [AT, Théorème II.2]).

Definition 3.1. Let $0<p<\infty$. The local Hardy space $\mathbf{h}^{\mathbf{p}}=\mathbf{h}^{\mathbf{p}}\left(\mathbb{R}_{+}, *(A)\right)$ is defined by

$$
\mathbf{h}^{\mathbf{p}}:=\left\{f \in \mathscr{S}_{1}^{\prime}\left(\mathbb{R}_{+}, *(A)\right): H_{0}^{+} f \in L^{p}\left(\mathbb{B}_{+}, A d x\right)\right\} .
$$

Moreover, we introduce the quasi-norm $\|f\|_{h^{p}}:=\left\|H_{0}^{+} f\right\|_{p, A}$ defining the topology on $\mathbf{h}^{\mathbf{p}}$.

We recall that for $1<p<\infty, \mathbf{h}^{\mathbf{p}}$ coincides with $L^{p}\left(\mathbb{R}_{+}, A d x\right)$. The elementary building blocks of $\mathbf{h}^{\mathbf{p}}$ are the (local) $(p, q, s)$-atoms. Assume throughout that the exponents $p$ and $q$ are admissible in the sense that $0<p \leq 1,1 \leq q \leq \infty$ and $p<q$, and put $s=[(2 \alpha+2)(1 / p-1)]$ where $[\beta]$ denotes the largest integer not exceeding $\beta$. 
DEFINITION 3.2. A (local) $(p, q, s)$-atom is a function $a \in L^{q}\left(\mathbb{R}_{+}, A d x\right)$ such that for some $x_{0} \in \mathbb{R}_{+}$and $r>0, \operatorname{supp}(a) \subset B\left(x_{0}, r\right)$ and

$$
\|a\|_{q, A} \leq m\left(B\left(x_{0}, r\right)\right)^{1 / q-1 / p}
$$

together with the following (local) moment condition: if $r$ can be chosen not exceeding 1 then

$$
\int_{0}^{\infty} a(x) x^{k} A(x) d x=0
$$

for all integers $k$ satisfying $0 \leq k \leq s$.

The following result characterizes $\mathbf{h}^{\mathbf{p}}$ in terms of atoms.

THEOREM 3.1 (see [BX4]). Let $0<p \leq 1$. Then $f \in \mathbf{h}^{\mathbf{p}}$ if and only if $f$ can be represented as a linear combination of $(p, q, s)$-atoms for any $1 \leq q \leq \infty, q>p$, that is

$$
f=\sum_{i} \lambda_{i} a_{i}
$$

where the $a_{i}$ are (local) $(p, q, s)$-atoms and $\sum_{i}\left|\lambda_{i}\right|^{p}<\infty$. Moreover, there exist positive constants $C_{1}$ and $C_{2}$ depending only on $p$ and $A$ such that

$$
C_{1}\left\{\sum_{i}\left|\lambda_{i}\right|^{p}\right\}^{1 / p} \leq\|f\|_{\mathrm{h}^{p}} \leq C_{2}\left\{\sum_{i}\left|\lambda_{i}\right|^{p}\right\}^{1 / p} .
$$

We now introduce the (local) molecules corresponding to the atoms defined above.

DEFINITION 3.3. For admissible components $p, q$ and $s$ and $\epsilon>\max \{s /(2 \alpha+$ 2 ), $1 / p-1$ ) set $a=1-1 / p+\epsilon$ and $b=1-1 / q+\epsilon$. A (local) $(p, q, s, \epsilon)$-molecule centred at $x_{0} \in \mathbb{R}_{+}$is a function $M \in L^{q}\left(\mathbb{R}_{+}, A d x\right)$ with $M(x)\left|B\left(x_{0},\left|x-x_{0}\right|\right)\right|^{b} \in$ $L^{q}\left(\mathbb{R}_{+}, A d x\right)$ satisfying the conditions

(i) $\|M\|_{q . A}^{a / b}\left\|M(x)\left|B\left(x_{0},\left|x-x_{0}\right|\right)\right|^{b}\right\|_{q, A}^{1-a / b}:=\mathscr{N}_{q}(M)<\infty$;

(ii) Let $\sigma$ be the positive number defined by $\left|B\left(x_{0}, \sigma\right)\right|^{1 / q-1 / p}=\|M\|_{q, A}$. If $\sigma<1$ then for any $R$ with $\sigma \leq R \leq 1$,

$$
\left|\int_{B\left(x_{0}, R\right)} M(x)\left(x-x_{0}\right)^{l} A(x) d x\right| \leq C_{A, l}\left(\frac{\sigma}{R}\right)^{\beta} R^{l}\left|B\left(x_{0}, R\right)\right|^{1-1 / p}
$$

for $l=0,1, \ldots, s$ where $\beta=\min \{a, s+2-1 / p\}$.

The following result gives the molecular characterization of $\mathbf{h}^{\mathbf{p}}$. 
THEOREM 3.2 (see [BX4]). Let $f \in \mathscr{S}_{1}^{\prime}\left(\mathbb{R}_{+}, *(A)\right)$. Then $f \in \mathbf{h}^{\mathbf{p}}$ if and only if it has a molecular decomposition

$$
f=\sum_{i} M_{i}
$$

where the $M_{i}$ are (local) $(p, q, s, \epsilon)$-molecules such that

$$
\sum_{i} \mathscr{N}_{q}\left(M_{i}\right)^{p}<\infty
$$

Moreover, if the above decomposition holds then

$$
\|f\|_{\mathrm{h}^{\mathrm{p}}} \sim \sum_{i} \mathscr{N}_{q}\left(M_{i}\right)^{p}
$$

For each $x \in \mathbb{R}_{+}$write $p_{x}(\lambda)=p(x, \lambda)$ and let $K_{x}$ denote the inverse Fourier transform of $p_{x}$ in the distributional sense. For each nonnegative integer $i$ define $K_{x}^{0}=K_{x}, K_{x}^{i}=\partial^{i} K_{x} / \partial x^{i}$ and denote by $p_{x}^{i}$ the Fourier transform of $K_{x}^{i}$. Then $p_{x}^{i}(\lambda)=\partial^{i} p(x, \lambda) / \partial x^{i}$. Choose an even $C^{\infty}$-function $\psi$ on $\mathbb{R}$ such that $\psi(x)=1$ for $|x| \leq 1 / 2$ and $\psi(x)=0$ for $|x| \geq 1$, and fix once and for all a kernel decomposition $K_{x}^{i}=\left(K_{x}^{i}\right)^{0}+\left(K_{x}^{i}\right)^{\infty}$ where $\left(K_{x}^{i}\right)^{0}=K_{x}^{i} \psi$ and $\left(K_{x}^{i}\right)^{\infty}=K_{x}^{i}(1-\psi)$.

Before giving some estimates for these kernels we define a variant of the class $S_{\eta, \delta}^{m}$.

DEFINITION 3.4. For nonnegative integers $i, N$ and $0<p \leq 2$ we say that a symbol $p(x, \lambda)$ belongs to the class $\mathscr{S}_{\eta \delta}^{m}(i, N, p)(0 \leq \eta, \delta \leq 1, m \in \mathbb{R})$ if $p(x, \lambda) \in S_{\eta, \delta}^{m}$ and for each $x \in \mathbb{R}_{+}, p_{x}$ extends to an even analytic function inside $\mathscr{F}_{\sigma}$ and the derivatives $p_{x}^{(j)}$ extend continuously to the whole of $\mathscr{F}_{\sigma}$, and $p(x, \lambda)$ satisfies

$$
\left|\frac{\partial^{j}}{\partial \lambda^{j}} \frac{\partial^{i}}{\partial x^{i}} p(x, \lambda)\right| \leq C_{i j}(1+|\lambda|)^{m-\eta j+\delta i}
$$

for $j=0,1, \ldots, N$, where $C_{i j}>0$ is independent of $x$.

The following estimates for the kernels $K_{x}^{i}$ can be proved similarly to [An, Proposition 5] and [BX4, Lemma 3.9].

LEMMA 3.1. Suppose that $\mu \geq(1-\eta)(2 \alpha+2)(1 / p-1 / 2)(0<\eta \leq 1,0<p \leq 1)$ and $p(x, \lambda) \in \mathscr{S}_{\eta 0}^{-\mu}(i, N, p)$ with $N>(2 \alpha+2) / p-\alpha-1+3 /(2 \eta)$. Then for each $x \in \mathbb{R}_{+}$and $0<R \leq 1,\left(K_{x}^{i}\right)^{\infty} \in L^{1}\left(\mathbb{R}_{+}, A d x\right)$ and

$$
\begin{gathered}
\left\|\left(K_{x}^{i}\right)^{\infty}\right\|_{1, A} \leq C_{A, i, N}, \\
\int_{0}^{R} y\left|\left(K_{x}^{i}\right)^{0}(y)\right| A(y) d y \leq C_{A, i, N} R
\end{gathered}
$$


and

$$
\int_{R}^{1} y^{-1}\left|\left(K_{x}^{i}\right)^{0}(y)\right| A(y) d y \leq C_{A, i, N} R^{-1}
$$

Let $\phi$ be an even nonnegative $C^{\infty}$-function supported in $\{x \in \mathbb{R}: 1 / 2<|x|<2\}$ and satisfying $\sum_{j=-\infty}^{\infty} \phi\left(2^{-j} x\right)=1$ for $x \neq 0$. Put $\phi_{j}(x)=\phi\left(2^{-j} x\right)$ for $j=1,2, \ldots$ and $\phi_{0}(x)=1-\sum_{j=1}^{\infty} \phi\left(2^{-j} x\right)$. For $p(x, \lambda) \in \mathscr{S}_{\eta 0}^{-\mu}(i, N, p)$ we fix once and for all a dyadic decomposition $p_{x}^{i}(\lambda)=\sum_{k=0}^{\infty} p_{x, k}^{i}(\lambda)$ where $p_{x, k}^{i}(\lambda)=p_{x}^{i}(\lambda) \phi_{k}(\lambda)$. Thus the corresponding decomposition for the kernel $K_{x}^{i}$ is $K_{x}^{i}(y)=\sum_{k=0}^{\infty} K_{x, k}^{i}(y)$ where $\widehat{K}_{x, k}^{i}(\lambda)=p_{x, k}^{i}(\lambda)$. Let $l_{x}^{i}(u)=\sum_{k=0}^{\infty} l_{x, k}^{i}(u)$ where $F_{0}\left(l_{x, k}^{i}\right)=p_{x, k}^{i}$.

Throughout the remainder of the paper we shall always assume that for each $x \in \mathbb{R}_{+}$, $p_{x}^{i}$ is rapidly decreasing (that is, $p_{x}^{i} \in \mathscr{S}\left(\mathscr{F}_{0}\right)$, the usual Schwartz space) although none of our estimates will depend upon the actual rate of decrease. It suffices to flatten $p_{x}^{i}$ or, equivalently, to regularize $K_{x}^{i}$ in the standard way. Thus by Theorem 1.3, $K_{x}^{i} \in \mathscr{S}_{2}\left(\mathbb{R}_{+}, *(A)\right)$.

For $p(x, \lambda) \in \mathscr{S}_{\eta 0}^{-\mu}(i, N, p)$ choose an even $C^{\infty}$-function $\omega^{0}$ such that

$$
\omega^{0}= \begin{cases}1, & |x| \leq 1 / 4 \\ 0, & |x| \geq 1 / 2\end{cases}
$$

For any positive integer $j$ and $x, r>0$ put $l_{x, k j}^{i}:=\left(1-\widetilde{\omega}_{j}\right) l_{x, k}^{i}, K_{x, k j}^{i}:=\mathscr{A}^{-1}\left(l_{x, k j}^{i}\right)$ and $p_{x, k j}^{i}:=F_{0}\left(l_{x, k j}^{i}\right)$ where $\widetilde{\omega}_{j}(x):=\omega^{0}\left(2^{-j} r^{-1} x\right)$. Observe that $l_{x, k}^{i}-l_{x, k j}^{i}$ is supported in $\left\{u:|u| \leq 2^{j} r\right\}$. Using properties of the Abel transform in [T1] we have

$$
K_{x, k}^{i}(y)=K_{x, k j}^{i}(y) \quad \text { if } x>2^{j-2} r .
$$

The following lemma is proved similarly to [BX4, Lemma 3.21].

LEMMA 3.2. Suppose that $p(x, \lambda) \in \mathscr{S}_{\eta 0}^{-\mu}(i, N, p)$ with $0<p \leq 1, \alpha \geq 0$ and $N>(2 \alpha+2) / p-\alpha-1+3 /(2 \eta)$. Given $j \in \mathbb{N}_{0}$ and $r>0$ such that $2^{j} r \leq 1$ we have for any nonnegative numbers $L_{1}$ and $L_{2}$ with $L_{2} \leq N$

$$
\left\{\int_{0}^{\infty}\left|p_{x, k j}^{i}(\lambda)(1+\lambda)^{L_{1}}\right|^{2} d \lambda\right\}^{1 / 2} \leq \begin{cases}C_{A, i, N}\left(2^{j} r\right)^{-L_{1}} 2^{k / 2}, & 2^{j+k} r<1 \\ C_{A, i, N}\left(2^{j} r\right)^{-L_{2}} 2^{k\left(L_{1}-L_{2}+1 / 2\right)}, & \text { otherwise }\end{cases}
$$

Let $a$ be a (local) $(p, \infty, s)$-atom supported in $B\left(x_{0}, r\right)$ with $x_{0} \in \mathbb{R}_{+}$and $0<r \leq 1$. For any positive integer $j \leq j_{0}+2$ put $\phi_{j, r}(x)=\phi\left(2^{-j} r^{-1}\left(x-x_{0}\right)\right), M_{1}(x)=$ $\sum_{j=-\infty}^{l} T a(x) \phi_{j, r}(x)$ and $M_{j}(x)=T a(x) \phi_{j, r}(x)(j \geq 2)$ where $\phi$ is defined as before and $j_{0}$ is the unique integer such that $1 \leq 2^{j_{0}} r<2$. 
THEOREM 3.3. Suppose that $p(x, \lambda) \in \mathscr{S}_{\eta 0}^{-\mu}(i, N, p)$ with $0<p \leq 1, \alpha \geq 0$, $N>(2 \alpha+2) / p-\alpha-1+3 /(2 \eta)$ and $i=0,1, \ldots, s+1$. Then for each integer $1 \leq j \leq j_{0}+2, M_{j}$ is a (local) $(p, 2, s, \epsilon)$-molecule and

$$
\sum_{j=1}^{j_{0}+2} \mathscr{N}_{2}\left(M_{j}\right) \leq C_{A, p} .
$$

PROOF. For each $x \in \mathbb{R}_{+}$fix a dyadic decomposition $p_{x}^{i}=\sum_{k=0}^{\infty} p_{x, k}^{i}$ and the corresponding decomposition $K_{x}^{i}=\sum_{k=0}^{\infty} K_{x, k}^{i}$ as before. By Definition 2.1, (3.1), (1.8) and (1.9) and using the moment condition of an atom we observe for $j=$ $2,3, \ldots, j_{0}+2$

$$
\begin{aligned}
M_{j}(x) & =\phi_{j, r}(x) \int_{0}^{\infty} a(y) T_{x} K_{x}(y) A(y) d y \\
& =\phi_{j, r}(x) \sum_{k=0}^{\infty} \int_{0}^{\infty} a(y) T_{x} K_{x, k}(y) A(y) d y \\
& =\phi_{j, r}(x) \sum_{k=0}^{\infty} \int_{0}^{\infty} a(y) T_{x} K_{x, k j}(y) A(y) d y \\
& =\int_{0}^{1}(1-u)^{s-1}\left\{\int_{0}^{\infty} a(y)\left(y-x_{0}\right)^{s} G_{j, x, u, s}(y) A(y) d y\right\} d u
\end{aligned}
$$

where

$$
G_{j, x, u, s}(y)= \begin{cases}\phi_{j, r}(x) \sum_{k=0}^{\infty}\left(T_{x} K_{x, k j}\right)^{(s)}\left(x_{0}+u\left(y-x_{0}\right)\right)-\left(T_{x} K_{x, k j}\right)^{(s)}\left(x_{0}\right), & s>0 \\ \phi_{j, r}(x) \sum_{k=0}^{\infty} T_{x} K_{x, k j}(y)-T_{x} K_{x, k j}\left(x_{0}\right), & s=0 .\end{cases}
$$

Put $\zeta_{y, u, x_{0}}=x_{0}+u\left(y-x_{0}\right)$. In view of Theorem 1.1 and Theorem 1.2 we have

$$
\begin{aligned}
\left|G_{j, x, u, s}(y)\right| & \leq \phi_{j, r}(x) \sum_{k=0}^{\infty} \int_{0}^{\infty}\left|p_{x, k j}(\lambda) \varphi_{\lambda}(x)\left(\varphi_{\lambda}^{(s)}\left(\zeta_{y, u, x_{0}}\right)-\varphi_{\lambda}^{(s)}\left(x_{0}\right)\right)\right||c(\lambda)|^{-2} d \lambda \\
= & \phi_{j, r}(x) \sum_{u\left|y-x_{0}\right| 2^{k} \leq 1} \int_{0}^{\infty}\left|p_{x, k j}(\lambda) \varphi_{\lambda}(x)\left(\varphi_{\lambda}^{(s)}\left(\zeta_{y, u, x_{0}}\right)-\varphi_{\lambda}^{(s)}\left(x_{0}\right)\right)\right||c(\lambda)|^{-2} d \lambda \\
& +\sum_{u\left|y-x_{0}\right| 2^{k}>1} \int_{0}^{\infty}\left|p_{x, k j}(\lambda) \varphi_{\lambda}(x)\left(\varphi_{\lambda}^{(s)}\left(\zeta_{y, u, x_{0}}\right)-\varphi_{\lambda}^{(s)}\left(x_{0}\right)\right)\right||c(\lambda)|^{-2} d \lambda \\
& :=J_{1}+J_{2} .
\end{aligned}
$$


Assume that $x_{0} \leq 2 r$. Then by the mean-value theorem, Theorem 1.2 and Lemma 1.3 and Lemma 3.2 (with $L_{1}=s+\alpha+2$ and $L_{2}=N$ )

$$
\begin{aligned}
J_{1} \leq & C_{A} u\left|y-x_{0}\right| \phi_{j, r}(x) \sum_{u\left|y-x_{0}\right| 2^{k} \leq 1} \int_{0}^{\infty}\left|p_{x, k j}(\lambda) \varphi_{\lambda}(x)(1+\lambda)^{s+1}\right||c(\lambda)|^{-2} d \lambda \\
= & C_{A} u\left|y-x_{0}\right| \phi_{j, r}(x) \sum_{u\left|y-x_{0}\right| 2^{k} \leq 1} \int_{0}^{1}\left|p_{x, k j}(\lambda) \varphi_{\lambda}(x)(1+\lambda)^{s+1}\right||c(\lambda)|^{-2} d \lambda \\
& +C_{A} u\left|y-x_{0}\right| \phi_{j, r}(x) \sum_{u\left|y-x_{0}\right| 2^{k} \leq 1} \int_{1}^{x^{-1}}\left|p_{x, k j}(\lambda) \varphi_{\lambda}(x)(1+\lambda)^{s+1}\right||c(\lambda)|^{-2} d \lambda \\
& +C_{A} u\left|y-x_{0}\right| \phi_{j, r}(x) \sum_{u\left|y-x_{0}\right| 2^{k} \leq 1} \int_{x^{-1}}^{\infty}\left|p_{x, k j}(\lambda) \varphi_{\lambda}(x)(1+\lambda)^{s+1}\right||c(\lambda)|^{-2} d \lambda \\
\leq & \left.C_{A} u\left|y-x_{0}\right| \phi_{j, r}(x) \sum_{u\left|y-x_{0}\right| 2^{k} \leq 1} A(x)^{-1 / 2}\left\{\int_{0}^{\infty}\left|p_{x, k j}(\lambda)(1+\lambda)^{s+\alpha+2}\right|^{2} d \lambda\right)\right\}^{1 / 2} \\
\leq & C_{A} u\left|y-x_{0}\right| \phi_{j, r}(x) A(x)^{-1 / 2}\left(2^{j} r\right)^{-s-\alpha-5 / 2} .
\end{aligned}
$$

Similarly, we obtain

$$
\begin{aligned}
J_{2} & \leq C_{A} \phi_{j . r}(x) \sum_{u\left|y-x_{0}\right| 2^{k}>1} \int_{0}^{\infty}\left|p_{x, k j}(\lambda) \varphi_{\lambda}(x)(1+\lambda)^{s}\right||c(\lambda)|^{-2} d \lambda \\
& \leq C_{A}\left(u\left|y-x_{0}\right|\right)^{N-s-\alpha-3 / 2} \phi_{j, r}(x) A(x)^{-1 / 2}\left(2^{j} r\right)^{-N} .
\end{aligned}
$$

For $x_{0}>2 r$ we argue similarly:

$$
J_{1} \leq \begin{cases}C_{A} u\left|y-x_{0}\right| \phi_{j, r}(x) A(x)^{-1 / 2}\left(2^{j} r\right)^{-s-\alpha-5 / 2}, & x_{0} \leq 2^{j+2} r ; \\ C_{A} u\left|y-x_{0}\right| \phi_{j, r}(x) A(x)^{-1}\left(2^{j} r\right)^{-s-2}, & \text { otherwise }\end{cases}
$$

and

$$
J_{2} \leq \begin{cases}C_{A}\left(u\left|y-x_{0}\right|\right)^{N-s-\alpha-3 / 2} \phi_{j, r}(x) A(x)^{-1 / 2}\left(2^{j} r\right)^{-N}, & x_{0} \leq 2^{j+2} r \\ C_{A}\left(u\left|y-x_{0}\right|\right)^{N-s-1} \phi_{j, r}(x) A(x)^{-1}\left(2^{j} r\right)^{-N}, & \text { otherwise. }\end{cases}
$$

Hence by Definition 3.2 and Lemma 1.1 we have for $j=2,3, \ldots, j_{0}+2$

$$
\left\|M_{j}\right\|_{2, A} \leq C_{A}\left|B\left(x_{0}, 2^{j} r\right)\right|^{1 / 2-1 / p} 2^{-\gamma j}
$$

where

$$
\gamma= \begin{cases}\min \left\{s+2 \alpha+3-\frac{2 \alpha+2}{p}, N-\frac{2 \alpha+2}{p}\right\}, & x_{0} \leq 2^{j+2} r \\ \min \{s+2-1 / p, N-1 / p\}, & \text { otherwise. }\end{cases}
$$


By Definition 3.2 and Theorem 2.1 we observe

$$
\left\|M_{1}\right\|_{2, A} \leq C_{A}\|a\|_{2, A} \leq C_{A}\left|B\left(x_{0}, r\right)\right|^{1 / 2-1 / p} .
$$

We now prove that the $M_{j}$ satisfy Definition 3.3(i) (with $q=2$ ). Using (3.4), (3.5) and Lemma 1.1 we obtain for $j=1,2, \ldots, j_{0}+2$

$$
\begin{aligned}
\left\|M_{j}(x)\left|B\left(x_{0},\left|x-x_{0}\right|\right)\right|^{b}\right\|_{2, A}^{1-a / b} & \leq C_{A, p}\left\|M_{j}\right\|_{2, A}^{1-a / b}\left|B\left(x_{0}, 2^{j} r\right)\right|^{b-a} \\
& \leq C_{A, p} 2^{\gamma(a / b-1) j}\left|B\left(x_{0}, 2^{j} r\right)\right|^{a / b(1 / p-1 / 2)} .
\end{aligned}
$$

Therefore by (3.4)-(3.6)

$$
\mathscr{N}_{2}\left(M_{j}\right) \leq C_{A, p} 2^{-\gamma j}, \quad j=1,2, \ldots, j_{0}+2 .
$$

It remains to show that the $M_{j}$ satisfy Definition 3.3(ii). We first observe that Theorem 2.1 and Definition 3.2 give

$$
\left|B\left(x_{0}, r\right)\right| \leq C_{A, q}\left|B\left(x_{0}, \sigma\right)\right| .
$$

If $R<2 r$ then we apply the Cauchy-Schwarz inequality, (3.8) and Lemma 1.1 to obtain

$$
\begin{aligned}
\left|\int_{B\left(x_{0}, R\right)} M_{j}(x)\left(x-x_{0}\right)^{l} A(x) d x\right| & \leq C_{A} R^{l}\|T a\|_{2, A}\left|B\left(x_{0}, R\right)\right|^{1 / 2} \\
& =C_{A} R^{l}\left|B\left(x_{0}, R\right)\right|^{1-1 / p}\left(\frac{\left|B\left(x_{0}, R\right)\right|}{\left|B\left(x_{0}, \sigma\right)\right|}\right)^{1 / p-1 / 2} \\
& \leq C_{A, p} R^{l}\left|B\left(x_{0}, R\right)\right|^{1-1 / p}\left(\frac{\sigma}{R}\right)^{\beta} .
\end{aligned}
$$

If $R \geq 2 r$ then write

$$
\begin{aligned}
\int_{B\left(x_{0}, R\right)} M_{j}(x)\left(x-x_{0}\right)^{l} A(x) d x= & \int_{B\left(x_{0}, R\right)} M_{j}(x) \psi_{R}(x)\left(x-x_{0}\right)^{l} A(x) d x \\
& +\int_{B\left(x_{0}, R\right)} M_{j}(x)\left(1-\psi_{R}(x)\right)\left(x-x_{0}\right)^{l} A(x) d x \\
:= & I_{R, j}^{(1)}+I_{R, j}^{(2)}
\end{aligned}
$$

where $\psi_{R}(x)=\psi\left(\left(x-x_{0}\right) / R\right)$. Let $E_{R}:=\left\{x \in \mathbb{R}_{+}: R / 2<\left|x-x_{0}\right|<R\right\}$. Then by (3.7) and Lemma 1.1

$$
\begin{aligned}
\left|I_{R, j}^{(2)}\right| & \leq C_{A} \int_{E_{R}}\left|M_{j}(x)\right|\left|x-x_{0}\right|^{l} A(x) d x \\
& \leq C_{A, p} R^{l}\left|E_{R}\right|^{1 / 2}\left|B\left(x_{0}, R\right)\right|^{-b}\left\|M_{j}(x)\left|B\left(x_{0},\left|x-x_{0}\right|\right)\right|^{b}\right\|_{2, A}
\end{aligned}
$$




$$
\begin{aligned}
& \leq C_{A, p} \mathscr{N}_{2}\left(M_{j}\right)^{b /(b-a)} R^{l}\left|B\left(x_{0}, R\right)\right|^{1 / 2-b}\left\|M_{j}\right\|_{2, A}^{a /(a-b)} \\
& \leq C_{A, p} 2^{-\gamma j} R^{l}\left|B\left(x_{0}, R\right)\right|^{1 / 2-b}\left|B\left(x_{0}, \sigma\right)\right|^{a} \\
& \leq C_{A, p} 2^{-\gamma j} R^{l}\left|B\left(x_{0}, R\right)\right|^{1-1 / p}\left(\frac{\sigma}{R}\right)^{\beta} .
\end{aligned}
$$

To estimate $I_{R, j}^{(1)}$ we use the Taylor expansion of $K_{x}(y)$ with respect to $x$ at $x=x_{0}$ to obtain

$$
\begin{aligned}
I_{R, j}^{(1)}= & \int_{B\left(x_{0}, R\right)} \tilde{\phi}_{j, r}(x) Q_{R, l}(x)\left(\int_{0}^{\infty} T_{x} a(y) K_{x}(y) A(y) d y\right) A(x) d x \\
= & \sum_{i=0}^{s} \frac{1}{i !} \int_{B\left(x_{0}, R\right)} \tilde{\phi}_{j, r}(x) Q_{R, l+i}(x)\left(\int_{0}^{\infty} T_{x} a(y) K_{x_{0}}^{i}(y) A(y) d y\right) A(x) d x \\
& +\frac{1}{(s+1) !} \int_{B\left(x_{0}, R\right)} \widetilde{\phi}_{j, r}(x) Q_{R, l+s+1}(x) \\
& \times\left(\int_{0}^{1}(1-u)^{s-1} \int_{0}^{\infty} T_{x} a(y) K_{\zeta_{x, u, x_{0}}}^{s+1}(y) A(y) d y d u\right) A(x) d x \\
:= & I_{1, j}+I_{2, j}
\end{aligned}
$$

where $Q_{R, l}=\psi_{R}(x)\left(x-x_{0}\right)^{l}, \zeta_{x, u, x_{0}}=x_{0}+u\left(x-x_{0}\right)$ and $\tilde{\phi}_{j, r}(x)=\sum_{k=0}^{l} \phi_{k, r}(x)$ if $j=1$ and $\widetilde{\phi}_{j, r}(x)=\phi_{j, r}(x)$ otherwise. Applying Lemma 3.1, [BX4, Lemma 3.11], (3.8) and Lemma 1.1 and arguing in the same way as in the proof of [BX4, Lemma 3.12] we obtain

$$
\left|I_{1, j}\right| \leq C_{A, p} R^{l}\left|B\left(x_{0}, R\right)\right|^{1-1 / p}\left(\frac{\sigma}{R}\right)^{\beta}
$$

It suffices to obtain the same estimates for $I_{2, j}$. If $j>1$ then by (3.1) we use the cancellation property and the Taylor expansion of $T_{x} K_{\zeta_{x, u, x_{0}}, k j}^{s+1}(y)$ about $y=x_{0}$ to obtain

$$
\begin{aligned}
I_{2, j}= & \frac{1}{(s+1) !} \sum_{k=0}^{\infty} \int_{B\left(x_{0}, R\right)} \tilde{\phi}_{j, r}(x) Q_{R, l+s+1}(x) \\
& \times\left(\int_{0}^{1}(1-u)^{s-1} \int_{0}^{\infty} a(y) T_{x} K_{\zeta_{x, k, x_{0}, k}+1}(y) A(y) d y d u\right) A(x) d x \\
= & \frac{1}{(s+1) !} \int_{B\left(x_{0}, R\right)} \tilde{\phi}_{j, r}(x) Q_{R, l+s+1}(x)\left(\int_{0}^{1}(1-u)^{s-1} \int_{0}^{1}(1-v)^{s-1}\right. \\
& \left.\times \int_{0}^{\infty} a(y)\left(y-x_{0}\right)^{s} \widetilde{G}_{j, x, u, v, s}(y) A(y) d y d v d u\right) A(x) d x
\end{aligned}
$$


where

$$
\widetilde{G}_{j, x, u, v, s}(y)= \begin{cases}\sum_{k=0}^{\infty}\left(\left(T_{x} K_{\zeta_{x, u, x_{0}, k j}}^{s+1}\right)^{(s)}\left(\zeta_{y, v, x_{0}}\right)-\left(T_{x} K_{\zeta_{x, u, x_{0}, k j}}^{s+1}\right)^{(s)}\left(x_{0}\right)\right), & s>0 ; \\ \sum_{k=0}^{\infty}\left(T_{x} K_{x, k j}^{1}(y)-T_{x} K_{x, k j}^{1}\left(x_{0}\right)\right), & s=0 .\end{cases}
$$

A similar argument to the proof of (3.4) gives for $j>1$

$$
\left|I_{2, j}\right| \leq \begin{cases}C_{A, p} R^{l+s+1}\left|B\left(x_{0}, R\right)\right|^{1-1 / p}\left(\frac{r}{R}\right)^{s+2 \alpha+3-(2 \alpha+2) / p}, & x_{0} \leq R ; \\ C_{A, p} R^{l+s+1}\left|B\left(x_{0}, R\right)\right|^{1-1 / p}\left(\frac{r}{R}\right)^{s+2-1 / p}, & x_{0}>R\end{cases}
$$

where $R \geq 2 r$.

We now consider the case when $j=1$. Assume $x_{0}<2 r$ and write

$$
\begin{aligned}
I_{2,1}= & \frac{1}{(s+1) !} \sum_{k=0}^{k_{0}} \int_{B\left(x_{0}, R\right)} \widetilde{\phi}_{j, r}(x) Q_{R, l+s+1}(x) \\
& \times\left(\int_{0}^{1}(1-u)^{s-1} \int_{0}^{\infty} a(y) T_{x} K_{\zeta_{x, u, x_{0}, k}+1}^{s+1}(y) A(y) d y d u\right) A(x) d x \\
& +\frac{1}{(s+1) !} \sum_{k=k_{0}}^{\infty} \int_{B\left(x_{0}, R\right)} \widetilde{\phi}_{j, r}(x) Q_{R, l+s+1}(x) \\
& \times\left(\int_{0}^{1}(1-u)^{s-1} \int_{0}^{\infty} a(y) T_{x} K_{\zeta_{x, \mu, x_{0}, k}}^{s+1}(y) A(y) d y d u\right) A(x) d x \\
:= & I_{2,1}^{(1)}+I_{2,1}^{(2)},
\end{aligned}
$$

where $k_{0}$ is the unique integer such that $1 \leq 2^{k_{0}} r<2$. Using Theorem 1.1, Lemma 1.1 and Definition 3.2 a straightforward calculation gives

$$
\left|I_{2,1}^{(1)}\right| \leq C_{A, p} r^{l+s+2 \alpha+3-(2 \alpha+2) / p} .
$$

For $l_{2,1}^{(2)}$ we observe

$$
\begin{aligned}
\left|I_{2,1}^{(2)}\right| \leq & C_{A, p}\left(\sum_{k=k_{0}}^{\infty} \int_{0}^{2^{-k+1}} \widetilde{\phi}_{j, r}(x) Q_{R, l+s+1}(x)\right. \\
& \times\left(\int_{0}^{1}(1-u)^{s-1} \int_{0}^{2^{-k+1}} a(y) T_{x} K_{\zeta_{x, u, x_{0}, k}^{s+1}}(y) A(y) d y d u\right) A(x) d x \\
& +\sum_{k=k_{0}}^{\infty} \int_{2^{-k+1}}^{3 r} \tilde{\phi}_{j, r}(x) Q_{R, l+s+1}(x)
\end{aligned}
$$




$$
\begin{aligned}
& \times\left(\int_{0}^{1}(1-u)^{s-1} \int_{0}^{2^{-k}} a(y) T_{x} K_{\zeta_{x, u, x_{0}, k}}^{s+1}(y) A(y) d y d u\right) A(x) d x \\
& +\sum_{k=k_{0}}^{\infty} \int_{0}^{2^{-k}} \widetilde{\phi}_{j, r}(x) Q_{R, l+s+1}(x) \\
& \times\left(\int_{0}^{1}(1-u)^{s-1} \int_{2^{-k+1}}^{3 r} a(y) T_{x} K_{\zeta_{x, u, x_{0}, k}}^{s+1}(y) A(y) d y d u\right) A(x) d x \\
& +\sum_{k=k_{0}}^{\infty} \int_{2^{-k}}^{3 r} \widetilde{\phi}_{j, r}(x) Q_{R, l+s+1}(x) \\
& \left.\times\left(\int_{0}^{1}(1-u)^{s-1} \int_{2^{-k}}^{3 r} a(y) T_{x} K_{\zeta_{x, u, x_{0}, k}}^{s+1}(y) A(y) d y d u\right) A(x) d x\right) \\
& :=C_{A, p}\left(J_{1}+J_{2}+J_{3}+J_{4}\right) .
\end{aligned}
$$

Applying Theorem 1.1, Lemma 1.1 and Definition 3.2 we obtain

$$
J_{i} \leq C_{A, p} r^{l+s+2 \alpha+3-(2 \alpha+2) / p}, \quad i=1,2,3 .
$$

Write

$$
\begin{aligned}
J_{4}= & \sum_{k=k_{0}}^{\infty} \sum_{j=k_{0}}^{k} \int_{2^{-k}}^{2^{-j-1}} \widetilde{\phi}_{j, r}(x) Q_{R, l+s+1}(x) \\
& \times\left(\int_{0}^{1}(1-u)^{s-1} \int_{2^{-j}}^{2^{-j+1}} a(y) T_{x} K_{\zeta_{x, u, x_{0}, k}+1}(y) A(y) d y d u\right) A(x) d x \\
& +\sum_{k=k_{0}}^{\infty} \sum_{j=k_{0}}^{k} \int_{2^{-j-1}}^{2^{-j+2}} \widetilde{\phi}_{j, r}(x) Q_{R, l+s+1}(x) \\
& \times\left(\int_{0}^{1}(1-u)^{s-1} \int_{2^{-j}}^{2^{-j+1}} a(y) T_{x} K_{\zeta_{x, u, x_{0}, k}+1}(y) A(y) d y d u\right) A(x) d x \\
& +\sum_{k=k_{0}}^{\infty} \sum_{j=k_{0}}^{k} \int_{2^{-j+2}}^{3 r} \widetilde{\phi}_{j, r}(x) Q_{R, l+s+1}(x) \\
& \times\left(\int_{0}^{1}(1-u)^{s-1} \int_{2^{-j}}^{2^{-j+1}} a(y) T_{x} K_{\zeta_{x, u, x_{0}, k}, k}^{s+1}(y) A(y) d y d u\right) A(x) d x \\
:= & J_{4}^{(1)}+J_{4}^{(2)}+J_{4}^{(3)} .
\end{aligned}
$$

To estimate $J_{4}^{(i)}(i=1,2,3)$ we introduce smooth cut-off functions as in [An, Section 2]. Let $\omega$ be an even $C^{\infty}$-function on $\mathbb{R}$ such that $\omega(u)=1$ for $|u| \leq 1 / 4$ and $\omega(u)=0$ for $|u| \geq 1 / 2$, and set $\omega_{j}(u)=\omega\left(2^{j} u\right)$ for each $j \in \mathbb{N}_{0}$. Then 
$\omega_{j}(u)=1$ for $|u| \leq 2^{-j-2}, \omega_{j}(u)=0$ for $|u| \geq 2^{-j-1}$ and $\left|d^{i} \omega_{j}(u) / d u^{i}\right| \leq C_{i} 2^{i j}$, $i=0,1,2, \ldots$. For each $x \in \mathbb{R}_{+}$denote by $l_{x}^{i}$ the inverse classical Fourier transform of $p_{x}^{i}$ as before. For a dyadic decomposition of $p_{x}^{i}$ let $l_{x}^{i}(u)=\sum_{k=0}^{\infty} l_{x, k}^{i}(u)$ be the corresponding decomposition where $F_{0} l_{x, k}^{i}(\lambda)=p_{x, k}^{i}\left(2^{-k} \lambda\right)$. Put $l_{x, k j}^{i}=\left(1-\omega_{j}\right) l_{x, k}^{i}$ and let $K_{x, k j}^{i}=\mathscr{A}^{-1}\left(l_{x, k j}^{i}\right)$ and $p_{x, k j}^{i}=F_{0}\left(l_{x, k j}^{i}\right)$. Then $l_{x, k}^{i}-l_{x, k j}^{i}$ is an even $C^{\infty}$-function supported in $\left[-2^{-j-1}, 2^{-j-1}\right]$, and hence using properties of the Abel transform (see [T1, Theorem 6.4]) we see that $K_{x, k}^{i}-K_{x, k j}^{i}=\mathscr{A}^{-1}\left(l_{x, k}^{i}-l_{x, k j}^{i}\right)$ is also supported in $\left[-2^{-j-1}, 2^{-j-1}\right]$. Consequently

$$
K_{x, k}^{i}(y)=K_{x, k j}^{i}(y), \quad y>2^{-j-1} .
$$

Arguing as in [An, Lemma 15] we have for $\beta_{1}<\beta_{2}$

$$
\left(\sum_{j=0}^{k}\left(2^{-\beta_{2} j}\left\|\left(1-\omega_{j}\right) l_{x, k}^{i}\right\|_{H_{2}^{\beta_{1}}}\right)^{2}\right)^{1 / 2} \leq C_{A} 2^{k\left(1 / 2+\beta_{1}-\beta_{2}\right)}
$$

where $H_{2}^{\beta}$ is the usual Sobolev space on $\mathbb{R}$. Thus applying Definition 3.2, Theorem 1.1, Theorem 1.2, Lemma 1.1, Lemma 1.2 and Lemma 1.3 and properties of the classical Fourier transform we obtain

$$
\begin{aligned}
\left|J_{4}^{(1)}\right| \leq & C_{A} r^{l+s+1-(2 \alpha+2) / p} \sum_{k=k_{0}}^{\infty} \sum_{j=k_{0}}^{k} \int_{2^{-k}}^{2^{-j-1}} \\
& \times\left(\int_{0}^{1} \int_{2^{-j}}^{2^{-j+1}}\left|T_{x} K_{\zeta_{x, u, x_{0}}, k j}^{s+1}(y)\right| A(y) d y d u\right) A(x) d x \\
\leq & C_{A} r^{l+s+1-(2 \alpha+2) / p} 2^{-(\alpha+1) j} \sum_{k=k_{0}}^{\infty} \sum_{j=k_{0}}^{k} \int_{2^{-k}}^{2^{-j-1}} \\
& \times\left(\int_{0}^{1}\left\{\int_{0}^{\infty}\left|T_{x} K_{\zeta_{x, u, x_{0}}, k j}^{s+1}(y)\right|^{2} A(y) d y\right\}^{1 / 2} d u\right) A(x) d x \\
\leq & C_{A} r^{l+s+1-(2 \alpha+2) / p} 2^{-(\alpha+1) j} \sum_{k=k_{0}}^{\infty} \sum_{j=k_{0}}^{k} \int_{2^{-k}}^{2^{-j-1}} \\
& \left.\times\left(\int_{0}^{1}\left\{\int_{0}^{\infty}\left|p_{\zeta_{x, \mu, x_{0}, k j}}^{s+1}(\lambda)\right|^{2}\right) d \lambda\right\}^{1 / 2} d u\right) A(x)^{1 / 2} d x \\
\leq & C_{A, p} r^{l+s+2 \alpha+3-(2 \alpha+2) / p} .
\end{aligned}
$$

Similarly, we have

$$
\left|J_{4}^{(i)}\right| \leq C_{A, p} r^{l+s+2 \alpha+3-(2 \alpha+2) / p}, \quad i=2,3,4 .
$$


Therefore, we have for $x_{0}<2 r$ and $R>2 r$

$$
\left|I_{2,1}\right| \leq C_{A, p} R^{l+s+1}\left|B\left(x_{0}, R\right)\right|^{1-1 / p}\left(\frac{r}{R}\right)^{s+2 \alpha+3-(2 \alpha+2) / p} .
$$

Arguing similarly we obtain for $x_{0} \geq 2 r$ and $R>2 r$

$$
\left|I_{2,1}\right| \leq \begin{cases}C_{A, p} R^{l+s+1}\left|B\left(x_{0}, R\right)\right|^{1-1 / p}\left(\frac{r}{R}\right)^{s+2 \alpha+3-(2 \alpha+2) / p}, & x_{0} \leq R \\ C_{A, p} R^{l+s+1}\left|B\left(x_{0}, R\right)\right|^{1-1 / p}\left(\frac{r}{R}\right)^{s+2-1 / p}, & x_{0}>R .\end{cases}
$$

In view of (3.8)-(3.10) we have

$$
\left|I_{1, j}\right| \leq C_{A, p} R^{l}\left|B\left(x_{0}, R\right)\right|^{1-1 / p}\left(\frac{\sigma}{R}\right)^{\beta}
$$

and the theorem is proved.

We have the following estimates for the derivatives of the heat kernel $h_{t}$.

LEMMA 3.3. For any nonnegative integer $k$ and $0<t \leq 1$ we have

$$
\left|h_{t}^{(k)}(x)\right| \leq \begin{cases}C_{A, k} t^{-k / 2-\alpha-1} e^{-\rho^{2} t}, & 0<x \leq c_{0} \sqrt{t} \\ C_{A, k} x^{-k-2 \alpha-2}\left(\frac{x^{2}}{t}\right)^{[k+2 \alpha+1]} e^{-\rho^{2} t} e^{-x^{2} /(4 t)}, & c_{0} \sqrt{t} \leq x \leq c_{1} \\ C_{A, k} x^{-1 / 2} A(x)^{-1 / 2}\left(\frac{x}{t}\right)^{[k+\alpha+1 / 2]+2} e^{-\rho^{2} t} e^{-x^{2} /(4 t)}, & x>c_{1}\end{cases}
$$

where $c_{0}$ and $c_{1}$ are positive constants.

ProOF. The lemma can be proved similarly to [BX2, Proposition 2.15] using Theorem 1.1, Theorem 1.2, Theorem 1.3, Lemma 1.1, Lemma 1.2 and Lemma 1.3.

For $0<t \leq 1$ define

$$
\tilde{p}_{t}(\lambda)=\int_{0}^{\infty} h_{t}(y) p(y, \lambda) \varphi_{\lambda}(y) A(y) d y .
$$

LEMMA 3.4. If $p(x, \lambda) \in \mathscr{S}_{\eta 0}^{-\mu}(i, N, p)$ with $N>(2 \alpha+2) / p-\alpha-1+3 /(2 \eta)$, $i=0$ and $0<p \leq 1$ then $\tilde{p}_{t}$ extends to an even analytic function inside $\mathscr{F}_{\sigma}$ and the derivatives $\tilde{p}_{t}^{(k)}$ extend continuously to the whole of $\mathscr{F}_{\sigma}$ and satisfy

$$
\left|\tilde{p}_{t}^{(k)}(x)\right| \leq C_{A, k}(1+|\lambda|)^{-k}, \quad \lambda \in \mathscr{F}_{\sigma}
$$

for $k=0,1, \ldots, N$. 
PROOF. The fact that $p(x, \lambda) \in \mathscr{S}_{\eta 0}^{-\mu}(i, N, p)$ with $i=0$ implies that $\tilde{p}_{\text {, }}$ extends to an even analytic function inside $\mathscr{F}_{\sigma}$ and the derivatives $\tilde{p}_{t}^{(k)}$ extend continuously to the whole of $\mathscr{F}_{\sigma}$. Write

$$
\begin{aligned}
\left|\tilde{p}_{i}^{(k)}(x)\right|= & \sum_{i=0}^{k}\left(\begin{array}{c}
k \\
i
\end{array}\right) \int_{0}^{\infty} h_{t}(y) \frac{\partial^{i}}{\partial \lambda^{i}} p(y, \lambda) \frac{\partial^{k-i}}{\partial \lambda^{k-i}} \varphi_{\lambda}(y) A(y) d y \\
= & \sum_{i=0}^{k}\left(\begin{array}{c}
k \\
i
\end{array}\right) \int_{0}^{\sqrt{t}} h_{t}(y) \frac{\partial^{i}}{\partial \lambda^{i}} p(y, \lambda) \frac{\partial^{k-i}}{\partial \lambda^{k-i}} \varphi_{\lambda}(y) A(y) d y \\
& +\sum_{i=0}^{k}\left(\begin{array}{l}
k \\
i
\end{array}\right) \int_{\sqrt{t}}^{1} h_{t}(y) \frac{\partial^{i}}{\partial \lambda^{i}} p(y, \lambda) \frac{\partial^{k-i}}{\partial \lambda^{k-i}} \varphi_{\lambda}(y) A(y) d y \\
& +\sum_{i=0}^{k}\left(\begin{array}{l}
k \\
i
\end{array}\right) \int_{1}^{\infty} h_{t}(y) \frac{\partial^{i}}{\partial \lambda^{i}} p(y, \lambda) \frac{\partial^{k-i}}{\partial \lambda^{k-i}} \varphi_{\lambda}(y) A(y) d y .
\end{aligned}
$$

Using Lemma 1.2, Lemma 3.3 and Lemma 1.1 we obtain for $\lambda \in \mathscr{F}_{\sigma}$ and $k=0$, $1, \ldots, N$

$$
\left|\tilde{p}_{t}^{(k)}(x)\right| \leq \begin{cases}C_{A, k}, & |\lambda| \leq 1 ; \\ C_{A, k}|\lambda|^{-k}, & 1<|\lambda| \leq \sqrt{t}^{-1} .\end{cases}
$$

For $\lambda \in \mathscr{F}_{\sigma}$ and $|\lambda|>\sqrt{t}^{-1}$ let $l=k / 2$ if $k$ is even and $l=(k+1) / 2$ otherwise. Then by (1.1), (1.6), Lemma 1.2, Lemma 1.3 and Lemma 3.3 we have

$$
\begin{aligned}
\left|\tilde{p}_{t}^{(k)}(x)\right|= & \sum_{i=0}^{k}\left(\begin{array}{c}
k \\
i
\end{array}\right) \int_{0}^{\infty} h_{t}(y) \frac{\partial^{i}}{\partial \lambda^{i}} p(y, \lambda) \frac{\partial^{k-i}}{\partial \lambda^{k-i}} \varphi_{\lambda}(y) A(y) d y \\
= & \sum_{i=0}^{k} \sum_{j=0}^{k-i}\left(\begin{array}{c}
k \\
i
\end{array}\right)\left(\begin{array}{c}
k-i \\
j
\end{array}\right) \int_{0}^{\infty} h_{t}(y) \frac{\partial^{i}}{\partial \lambda^{i}} p(y, \lambda) \\
& \times L_{y}^{l}\left(\left(\left(\lambda^{2}+\rho^{2}\right)^{-l}\right)^{(j)} \frac{\partial^{k-i}}{\partial \lambda^{k-i}} \varphi_{\lambda}(y)\right) A(y) d y \\
= & \sum_{i=0}^{k} \sum_{j=0}^{k-i}\left(\begin{array}{c}
k \\
i
\end{array}\right)\left(\begin{array}{c}
k-i \\
j
\end{array}\right)\left(\left(\lambda^{2}+\rho^{2}\right)^{-l}\right)^{(j)} \\
& \times \int_{0}^{\infty} L_{y}^{l}\left(h_{t}(y) \frac{\partial^{i}}{\partial \lambda^{i}} p(y, \lambda)\right) \frac{\partial^{k-i}}{\partial \lambda^{k-i}} \varphi_{\lambda}(y) A(y) d y .
\end{aligned}
$$

We now apply Lemma 1.1, Lemma 1.2, Lemma 1.3, Lemma 2.2 and Lemma 3.3 to obtain for $\lambda \in \mathscr{F}_{\sigma},|\lambda|>\sqrt{t}^{-1}$ and $k=0,1, \ldots, N$

$$
\left|\int_{0}^{\infty} L_{y}^{l}\left(h_{t}(y) \frac{\partial^{i}}{\partial \lambda^{i}} p(y, \lambda)\right) \frac{\partial^{k-i}}{\partial \lambda^{k-i}} \varphi_{\lambda}(y) A(y) d y\right|
$$




$$
\begin{aligned}
\leq & C_{A, k} \int_{0}^{\sqrt{i}}\left|L_{y}^{l}\left(h_{t}(y) \frac{\partial^{i}}{\partial \lambda^{i}} p(y, \lambda)\right) \frac{\partial^{k-i}}{\partial \lambda^{k-i}} \varphi_{\lambda}(y)\right| A(y) d y \\
& +C_{A, k} \int_{\sqrt{t}}^{1}\left|L_{y}^{l}\left(h_{t}(y) \frac{\partial^{i}}{\partial \lambda^{i}} p(y, \lambda)\right) \frac{\partial^{k-i}}{\partial \lambda^{k-i}} \varphi_{\lambda}(y)\right| A(y) d y \\
& +C_{A, k} \int_{1}^{\infty}\left|L_{y}^{l}\left(h_{t}(y) \frac{\partial^{i}}{\partial \lambda^{i}} p(y, \lambda)\right) \frac{\partial^{k-i}}{\partial \lambda^{k-i}} \varphi_{\lambda}(y)\right| A(y) d y \\
\leq & C_{A, k}(\sqrt{t})^{k-2 l-i-j}|\lambda|^{-i} .
\end{aligned}
$$

Hence for $\lambda \in \mathscr{F}_{\sigma},|\lambda|>\sqrt{t}^{-1}$ and $k=0,1, \ldots, N$

$$
\left|\tilde{p}_{t}^{(k)}(x)\right| \leq C_{A, k}|\lambda|^{-k}
$$

and this completes the proof of the lemma.

Let $\widetilde{K}_{t}$ denote the inverse Fourier transform of $\tilde{p}_{t}$ in the distributional sense.

LEMMA 3.5. Suppose that $p(x, \lambda) \in \mathscr{S}_{n 0}^{-\mu}(i, N, p)$ with $i=0, N>(2 \alpha+2) / p-$ $\alpha-1+3 /(2 \eta)$ and $0<p \leq 1$. Then for $0<t \leq 1$ and $|x-y| \geq 2$

$$
\left|T_{x}\left(\widetilde{K}_{t}\right)(y)\right| \leq C_{A, p}|x-y|^{-N} A(x)^{-1 / 2} A(y)^{-1 / 2} e^{-\sigma \rho|x-y|}
$$

where $\sigma=2 / p-1$.

PROOF. The lemma is proved in the same way as in [BX4, Lemma 3.15] using Lemma 3.4.

Let $\omega$ be a $C^{\infty}$-function on $\mathbb{R}$ such that $\omega(u)=0$ for $u \leq 1 / 2$ and $\omega(u)=1$ for $u \geq 1$. For any integer $j>1$ define an even $C^{\infty}$-function $\omega_{j}$ by

$$
\omega_{j}(u)=\omega(2(u+j-1)) \omega(2(-u+j-1))
$$

For each $x \in \mathbb{R}_{+}$let $p_{x}(\lambda)=p(x, \lambda)$ and let $K_{x}$ denote the inverse Fourier transform of $p_{x}$ as before. Denote by $l_{x}$ the Abel transform of $K_{x}$. Put $l_{x, j}=\left(1-\omega_{j}\right) l_{x}, p_{x, j}=$ $F_{0}\left(l_{x, j}\right)$ and $K_{x, j}=\mathscr{A}^{-1}\left(l_{x, j}\right)$. Since $l_{x}-l_{x, j}$ is supported in $[-j+5 / 4, j-5 / 4]$, by properties of the Abel transform in [T1, Théorème 6.4] we see that $K-K_{j}$ is also supported in $[-j+5 / 4, j-5 / 4]$ and hence

$$
K_{x}(u)=\widetilde{K}_{x, j}(u), \quad \text { if } u>j-\frac{5}{4}
$$

The proof of the following result is similar to that of [BX4, Lemma 3.19]. 
LEMMA 3.6. Suppose that $p(x, \lambda) \in \mathscr{S}_{\eta 0}^{-\mu}(i, N, p)$ with $i=0,0<p \leq 1$ and $N>(2 \alpha+2) / p-\alpha-1+3 /(2 \eta)$. Then for any $L$ with $0 \leq L<N-1 / 2$

$$
\left\{\int_{0}^{\infty}\left|p_{x, j}(\lambda)(1+\lambda)^{L}\right|^{2} d \lambda\right\}^{1 / 2} \leq C_{A, p} j^{-N} e^{-\sigma \rho j}, j=2,3, \ldots
$$

Finally we give $h^{p}$-estimates of the pseudo differential operators of class $L_{\eta, \delta}^{m}$.

THEOREM 3.4. Suppose that for $i=0,1, \ldots, N, p(x, \lambda) \in \mathscr{S}_{\eta 0}^{-\mu}(i, N, p)$ with $N>(2 \alpha+2) / p-\alpha-1+3 /(2 \eta)$ and $0<p \leq 1$. Then the pseudo differential operator $T$ given in Definition 2.1 is bounded on $\mathbf{h}^{\mathbf{p}}$.

PROOF. We refer to the proof of [BX4, Theorem 3.22]. By Theorem 3.1 we are reduced to showing that for any (local) $(p, \infty, s)$-atom $a$

$$
\left\|T_{a}\right\|_{\mathbf{h}^{p}} \leq C_{A, p}
$$

where the constant $C_{A, p}$ is independent of $a$. Suppose that $a$ is supported in $B\left(x_{0}, r\right)$ with $x_{0} \in \mathbb{R}_{+}$and $r>0$. Observe

$$
T a * h_{t}(x)=a * \widetilde{K}_{t}(x)
$$

and

$$
T a(x)=\int_{0}^{\infty} a(y) T_{x} K_{x}(y) A(y) d y .
$$

If $r>1$ then we write

$$
\begin{aligned}
\left\|H_{0}^{+}(T a)\right\|_{\mathrm{h}^{\mathrm{p}}}^{p}= & \int_{0}^{x_{0}+r+2}\left|H_{0}^{+}(T a)(x)\right|^{p} A(x) d x \\
& +\int_{x_{0}+r+2}^{\infty}\left|H_{0}^{+}(T a)(x)\right|^{p} A(x) d x \\
:= & I_{1}+I_{2} .
\end{aligned}
$$

Note that both $H_{0}^{+}$and $T$ are $L^{2}$-bounded and any $(p, \infty, s)$-atom must be a $(p, q, s)$ atom for all $q>p, 1 \leq q<\infty$. Applying Hölder's inequality and Lemma 1.1 then

$$
\begin{aligned}
I_{1} & \leq C_{A, p}\left\|H_{0}^{+}(T a)\right\|_{2, A}^{p} e^{2 \rho\left(x_{0}+r+2\right)(1-p / 2)} \\
& \leq C_{A, p}\|a\|_{2, A}^{p} e^{2 \rho\left(x_{0}+r+2\right)(1-p / 2)} \leq C_{A, p} .
\end{aligned}
$$


For $y \in B\left(x_{0}, r\right)$ and $x>x_{0}+r+2$ we have $x-y>2$. Hence by (3.13), Lemma 3.5 and Lemma 1.1 we have for $x>x_{0}+r+2$ and $0<t \leq 1$

$$
\left|T a * h_{t}(x)\right| \leq C_{A, p} A(x)^{-1 / p}\left(x-x_{0}-r\right)^{-N} .
$$

Consequently

$$
I_{2} \leq C_{A, p} \int_{x_{0}+r+2}^{\infty}\left(x-x_{0}-r\right)^{-N p} d x \leq C_{A, p}
$$

and (3.12) follows for $r>1$.

We now assume $r \leq 1$. As before let $\psi$ be an even $C^{\infty}$-function such that $\psi(x)=1$ if $|x| \leq 1 / 2$ and $\psi(x)=0$ if $|x| \geq 1$, and $\phi$ an even nonnegative $C^{\infty}$. function supported in $\{x \in \mathbb{R}: 1 / 2<|x|<2\}$ and satisfying $\sum_{j=-\infty}^{\infty} \phi\left(2^{-j} x\right)=1$ if $x \neq 0$. Write

$$
\begin{aligned}
T a(x) & :=\sum_{j=-\infty}^{\infty} T a(x) \phi_{j, r}(x) \tilde{\psi}(x)+T a(x)(1-\tilde{\psi}(x)) \\
& :=(T a)_{1}(x)+(T a)_{2}(x)
\end{aligned}
$$

where $\phi_{j, r}(x)=\phi\left(2^{-j} r^{-1}\left(x-x_{0}\right)\right)$ and $\tilde{\psi}(x)=\psi\left(\left(x-x_{0}\right) / 4\right)$. By Theorem 3.3 we see immediately that $(T a)_{1}$ is a $(p, 2, s, \epsilon)$-molecule and

$$
\mathscr{N}\left((T a)_{1}\right) \leq C_{A, p} .
$$

We now prove that $(T a)_{2}$ has an atomic decomposition. For each $j=2,3, \ldots$ let $Q_{j}=\left\{x \in \mathbb{R}_{+}: j<\left|x-x_{0}\right| \leq j+1\right\}$. Note that $(T a)_{2}(x)=0$ if $\left|x-x_{0}\right| \leq 2$. Hence

$$
(T a)_{2}(x)=\sum_{j=2}^{\infty}(T a)_{2}(x) \chi_{Q_{j}}(x):=\sum_{j=2}^{\infty} b_{j}(x) .
$$

Using (3.14), (3.11), (1.8) and (1.9) together with the cancellation property of an atom we observe for $x \in Q_{j}$

$$
\begin{aligned}
\operatorname{Ta}(x) & =\int_{0}^{\infty} a(y) T_{x} \widetilde{K}_{x, j}(y) A(y) d y \\
& =\int_{0}^{\infty} a(y)\left(y-x_{0}\right)^{s} \int_{0}^{1}(1-u)^{s-1} F_{j, x, u, s}(y) d u A(y) d y \\
& =\int_{0}^{1}(1-u)^{s-1} \int_{0}^{\infty} a(y)\left(y-x_{0}\right)^{s} F_{j, x, u, s}(y) A(y) d y d u
\end{aligned}
$$


if $s>0$, and

$$
T a(x)=\int_{0}^{\infty} a(y) F_{j, x, u, s}(y) A(y) d y
$$

if $s=0$, where

$$
F_{j, x, u, s}(y)= \begin{cases}\left(T_{x} \widetilde{K}_{x, j}\right)^{(s)}\left(x_{0}+u\left(y-x_{0}\right)\right)-\left(T_{x} \widetilde{K}_{x, j}\right)^{(s)}\left(x_{0}\right), & s>0 \\ T_{x} \widetilde{K}_{x, j}(y)-T_{x} \widetilde{K}_{x, j}\left(x_{0}\right), & s=0 .\end{cases}
$$

Thus applying Theorem 1.1, Theorem 1.2, Lemma 1.1, Lemma 1.2, Lemma 1.3 and Lemma 3.5 and arguing similarly as in the proof of Theorem 3.3 (see the estimate of $G_{j, x, u, s}(y)$ on page 16$)$ we obtain

$$
\left|F_{j, x, u, s}(y)\right| \leq \begin{cases}C_{A} j^{-N} e^{-\sigma \rho j} u\left|y-x_{0}\right| A(x)^{-1 / 2}, & x_{0} \leq 2 \\ C_{A} j^{-N} e^{-\sigma \rho j} u\left|y-x_{0}\right| A\left(x_{0}\right)^{-1 / 2} A(x)^{-1 / 2}, & x_{0}>2\end{cases}
$$

and hence by Definition 3.2

$$
\left|b_{j}(x)\right| \leq \begin{cases}C_{A, p} j^{-N} e^{-\sigma \rho j} r^{s+1}\left|B\left(x_{0}, r\right)\right|^{1-1 / p} \chi_{Q_{j}}(x) A(x)^{-1 / 2}, & x_{0} \leq 2 ; \\ C_{A, p} j^{-N} e^{-\sigma \rho j} r^{s+1}\left|B\left(x_{0}, r\right)\right|^{1-1 / p} \chi_{Q_{j}}(x) A\left(x_{0}\right)^{-1 / 2} A(x)^{-1 / 2}, & x_{0} \leq 2 .\end{cases}
$$

Thus by Lemma 1.1

$$
\left\|b_{j}\right\|_{2, A} \leq C_{A, p} j^{-N}\left|B\left(x_{0}, j+1\right)\right|^{1 / 2-1 / p} .
$$

Observe that $\operatorname{supp}\left(b_{j}\right) \subset B\left(x_{0}, j+1\right)$. Therefore, $a_{j}:=C_{A, p}^{-1} j^{N} b_{j}$ is a (local) $(p, 2, s)$-atom for each $j=2,3, \ldots$ and

$$
\left\|(T a)_{2}\right\|_{\mathrm{h}^{\mathrm{p}}} \leq C_{A, p} .
$$

The estimate (3.12) now follows from (3.15), (3.16) and Theorem 3.2, and this completes the proof of the theorem.

\section{References}

[An] J.-Ph. Anker, ' $l p$ Fourier multipliers on Riemannian symmetric spaces of the noncompact type', Ann. of Math. 132 (1990), 597-628.

[AT] A. Achour and K. Trimèche, 'La $g$-fonction de Littlewood-Paley associée à un opérateur différentiel singulier sur $(0, \infty)^{\prime}$, Ann. Inst. Fourier (Grenoble) 33 (1983), 203-226.

[BH] W. R. Bloom and H. Heyer, Harmonic analysis of probability measures on hypergroups, de Gruyter Studies in Math. 20 (de Gruyter, Berlin, 1995). 
[BX1] W. R. Bloom and Z. Xu, 'The Hardy-Littlewood maximal function for Chébli-Trimèche hypergroups', in: Applications of hypergroups and related measure algebras (Joint Summer Research Conference, Seattle, 1993), Contemporary Math. 183 (Amer. Math. Soc., Providence, 1995) pp. 45-70.

[BX2] - 'Maximal functions on Chébli-Trimèche hypergroups', preprint.

[BX3] - 'Fourier transforms of Schwartz functions on Chébli-Trimèche hypergroups', Monatsh. Math. 125 (1998), 89-109.

[BX4] - 'Fourier multipliers for local Hardy spaces on Chébli-Trimèche hypergroups', Canad. J. Math. 50 (1998), 897-928.

[CV] A. P. Calderón and R. Vaillancourt, 'A class of bounded pseudo-differential operators', Proc. Nat. Acad. Sci. U.S.A. 69 (1972), 1185-1187.

[F] C. Fefferman, ' $l$ pounds for pseudo-differential operators', Israel J. Math. 14 (1973), $413-417$

[G] D. Goldberg, 'A local version of real Hardy space', Duke Math. J. 46 (1979), $27-41$.

[H] L. Hörmander, Pseudo-differential operators and hypoelliptic equations, Proc. Sympos. Pure Math. 10 (Amer. Math. Soc., Providence, 1966), 138-183.

[K] T. Kawazoe, 'Atomic Hardy spaces on semisimple Lie groups', Japan. J. Math. 11 (1985), 293-343.

[PS] L. Päivärinta and E. Somersalo, 'A generalization of the Calderón-Vaillancourt theorem to $L^{p}$ and $h^{p}$, Math. Nachr. 138 (1988), 145-156.

[T1] K. Trimèche, 'Transformation intégrale de Weyl et théorème de Paley-Wiener associés à un opérateur différentiel singulier sur $(0, \infty)^{\prime}$, J. Math. Pures Appl. (9) 60 (1981), 51-98.

[T2] , 'Inversion of the lions transmutation operators using generalized wavelets', Appl. Comput. Harmon. Anal. 4 (1997), 97-112.

Division of Science and Engineering

Murdoch University

Perth WA 6150

Australia

e-mail: bloom@murdoch.edu.au

e-mail: zengfu@starwon.com.au 